TRANSACTIONS OF THE

AMERICAN MATHEMATICAL SOCIETY

Volume 364, Number 9, September 2012, Pages 4683-4712

S 0002-9947(2012)05467-5

Article electronically published on April 18, 2012

\title{
ON THE RESTRICTED VERMA MODULES AT THE CRITICAL LEVEL
}

\author{
TOMOYUKI ARAKAWA AND PETER FIEBIG
}

\begin{abstract}
We study the restricted Verma modules of an affine Kac-Moody algebra at the critical level with a special emphasis on their Jordan-Hölder multiplicities. Feigin and Frenkel conjectured a formula for these multiplicities that involves the periodic Kazhdan-Lusztig polynomials. We prove this conjecture for all subgeneric blocks and for the case of anti-dominant simple subquotients.
\end{abstract}

\section{INTRODUCTION}

The representation theory of affine Kac-Moody algebras at the critical level is one of the essential ingredients in the approach towards the geometric Langlands conjectures proposed by Beilinson and Drinfeld (cf. [BD96]). In particular, the correspondence between the center of the (completed) universal enveloping algebra of an affine Kac-Moody algebra at the critical level and the geometry of the space of operators associated with the Langlands dual datum (cf. [FF92]) is one of the main tools used in the construction of a part of the Langlands correspondence in BD96.

1.1. The local geometric Langlands conjectures. In [FG06] Frenkel and Gaitsgory formulated the local geometric Langlands conjectures, which relate the critical level representation theory of an affine Kac-Moody algebra to the geometry of an affine flag manifold. In a series of subsequent papers, the authors proved parts of these conjectures. In particular, in the paper [FG07] a derived equivalence between a certain category of $D$-modules on the affine flag variety and a derived version of the affine category $\mathcal{O}$ at the critical level was constructed using a localization functor of Beilinson-Bernstein type. It seems, however, hard to control the action of this equivalence on the respective hearts of the triangulated categories, and hence it is not yet possible to deduce information on the simple critical characters of the category $\mathcal{O}$ from the Frenkel-Gaitsgory result.

1.2. The Andersen-Jantzen-Soergel approach. In this paper we study the critical representation theory by a very different method that was inspired by results of Jantzen (cf. JJan79]) and Soergel (cf. [Soe90]) in the case of finite-dimensional complex Lie algebras and of Andersen, Jantzen and Soergel in the case of modular

Received by the editors June 4, 2010 and, in revised form, September 17, 2010.

2010 Mathematics Subject Classification. Primary 17B67; Secondary 81R10.

The first author was partially supported by the JSPS Grant-in-Aid for Scientific Research (B) No. 20340007.

The second author was supported by a grant of the Landesstiftung Baden-Württemberg.

(C)2012 American Mathematical Society

Reverts to public domain 28 years from publication 
Lie algebras and quantum groups (cf. AJS94]). The analogous results for the non-critical blocks of $\mathcal{O}$ for a symmetrizable Kac-Moody algebra can be found in [Fie06.

The main idea of this approach is to first describe the generic and subgeneric blocks of the respective representation theory in as much detail as possible, and then to deform an arbitrary block in such a way that it can be viewed as an intersection of generic and subgeneric blocks. This intersection procedure should then be described using only some underlying combinatorial datum (such as, for example, the associated integral Weyl group).

As a result we hope to be able to construct equivalences between various categories or links to categories defined in topological terms in the framework of the geometric Langlands program, and to deduce information on the respective simple characters. More specifically, we hope to find a correspondence between certain intersection cohomology sheaves on the Langlands dual affine flag variety and critical representations.

This paper provides the first steps in the approach described above. Its main result is the calculation of the simple characters in the subgeneric critical blocks. In order to explain our result, let us consider the respective categories in more detail.

1.3. Critical representations of affine Kac-Moody algebras. Let us denote by $\widehat{\mathfrak{g}}$ an affine Kac-Moody algebra and by $\widehat{\mathfrak{h}} \subset \widehat{\mathfrak{g}}$ its Cartan subalgebra (for the specialists it should be noted here that we consider the central extension of a loop algebra together with the grading operator). The one-dimensional center of $\widehat{\mathfrak{g}}$ acts semi-simply on each module in the category $\mathcal{O}$. Accordingly, each block of the category $\mathcal{O}$, i.e. each of its indecomposable direct summands, determines a central character. There is one such character, called the critical character, which is distinguished in more than one respect.

In this paper we focus on the following feature of the critical blocks. Let $\widehat{\mathfrak{h}}^{\star}$ be the dual space of the Cartan subalgebra and denote by $\delta \in \widehat{\mathfrak{h}}^{\star}$ the smallest positive imaginary root. Then the corresponding simple highest weight module $L(\delta)$ is of dimension one, and the tensor product $\cdot \otimes_{\mathbb{C}} L(\delta)$ defines a shift functor $T$ on $\mathcal{O}$ that is an equivalence. Now the critical blocks are exactly those that are preserved by the functor $T$. This allows us to consider, for each critical block, the corresponding graded center $\mathcal{A}$ (see Section 4.3).

The graded center is huge and intimately related to the center of the (completed) universal enveloping algebra at the critical level, which was determined in the fundamental work of Feigin and Frenkel (cf. [FF92]) (conjecturally, $\mathcal{A}$ is a quotient of the latter center). We use the results of Feigin-Frenkel to describe the action of $\mathcal{A}$ on the Verma modules contained in the critical blocks.

1.4. The restricted Verma modules. Let $\Delta(\lambda)$ be the Verma module with highest weight $\lambda \in \widehat{\mathfrak{h}}^{\star}$. We define the restricted Verma module $\bar{\Delta}(\lambda)$ with highest weight $\lambda$ as the quotient of $\Delta(\lambda)$ by the ideal of $\mathcal{A}$ generated by the homogeneous constituents $\mathcal{A}_{n}$ with $n \neq 0$. For our approach, the restricted Verma modules, not the ordinary ones, should be considered as the "standard objects" in the critical blocks.

We denote the irreducible quotient of $\Delta(\lambda)$ by $L(\lambda)$. Results of Frenkel and Feigin-Frenkel yield the characters of the restricted Verma modules, and the knowledge of the character of $L(\lambda)$ for all $\lambda$ is equivalent to the knowledge of the JordanHölder multiplicities $[\bar{\Delta}(\lambda): L(\mu)]$ for all pairs $\lambda, \mu$ of critical weights. 
1.5. The Feigin-Frenkel conjecture. Let us choose a critical indecomposable block $\mathcal{O}_{\Lambda}$ of $\mathcal{O}$ and let us identify the index $\Lambda$ with the subset of highest weights of the simple modules in $\mathcal{O}_{\Lambda}$. Since $\Lambda$ is critical we have $\lambda+\delta \in \Lambda$ if and only if $\lambda \in \Lambda$.

Let $\widehat{\mathcal{W}}$ be the affine Weyl group associated with our data, and let $\mathcal{W} \subset \widehat{\mathcal{W}}$ be the finite Weyl group. The integral Weyl group $\widehat{\mathcal{W}}(\Lambda)$ corresponding to $\Lambda$ is generated by the reflections with respect to those real roots that satisfy a certain integrality condition with respect to $\Lambda$. In the critical case, $\widehat{\mathcal{W}}(\Lambda)$ is the affinization of the corresponding finite integral Weyl group $\mathcal{W}(\Lambda) \subset \mathcal{W}$.

We define $\lambda \in \Lambda$ to be dominant, resp. anti-dominant, if it is dominant, resp. antidominant, with respect to the action of $\mathcal{W}(\Lambda)$, i.e. if it is the highest, resp. smallest, element in its $\mathcal{W}(\Lambda)$-orbit. We say that $\lambda$ is regular if it is regular with respect to $\mathcal{W}(\Lambda)$ (note that here we only refer to the finite integral Weyl group).

As mentioned before, $\widehat{\mathcal{W}}(\Lambda)$ is the affinization of $\mathcal{W}(\Lambda)$. In Lus80 Lusztig associated with a pair $w, x \in \widehat{\mathcal{W}}(\Lambda)$ the periodic polynomial $p_{x, w} \in \mathbb{Z}[v]$ (in Lusztig's paper these polynomials were indexed not by affine Weyl group elements, but by alcoves; see Section 4.5 for more details). The Feigin-Frenkel conjecture is the following.

Conjecture 1.1. Let $\Lambda$ be a critical equivalence class.

(1) The restricted linkage principle: For $\lambda, \mu \in \Lambda$ we have $[\bar{\Delta}(\lambda): L(\mu)]=0$ unless $\lambda$ and $\mu$ are contained in the same $\widehat{\mathcal{W}}(\Lambda)$-orbit.

(2) The restricted Verma multiplicities: Suppose that $\lambda \in \Lambda$ is regular and dominant. Under some further regularity conditions on $\Lambda$ (cf. Conjecture 4.8), we have

$$
[\bar{\Delta}(w \cdot \lambda): L(x . \lambda)]=p_{w, x}(1)
$$

for all $w, x \in \widehat{\mathcal{W}}(\Lambda)$.

The Feigin-Frenkel conjecture fits very well into a broader picture that was anticipated by Lusztig in his ICM address in 1990 in Kyoto (cf. Lus91]). There, Lusztig conjecturally linked the representation theory of modular Lie algebras, of quantum groups and of critical level representations of an affine Kac-Moody algebra to the topology of semi-infinite flag manifolds.

In AF09 we use the results of the present article in order to prove part (1) of the Feigin-Frenkel conjecture, the restricted linkage principle.

1.6. Our main result. Let $\mathfrak{h} \subset \widehat{\mathfrak{h}}$ be the finite part of the Cartan subalgebra and let us denote by $\lambda \mapsto \bar{\lambda}$ the corresponding restriction map from $\widehat{\mathfrak{h}}^{\star}$ to $\mathfrak{h}^{\star}$. We call a critical class $\Lambda$ subgeneric if its image $\bar{\Lambda}$ in $\mathfrak{h}^{\star}$ contains precisely two elements. Then we can define, following AJS94, a bijection $\alpha \uparrow:: \Lambda \rightarrow \Lambda$. Here, $\alpha$ denotes the unique positive finite root with $\bar{\Lambda}=\left\{\bar{\lambda}, s_{\alpha} \cdot \bar{\lambda}\right\}$. Here is our result:

Theorem 1.2. (1) If $\nu \in \Lambda$ is anti-dominant, then we have for all $\gamma \in \Lambda$

$$
[\bar{\Delta}(\gamma): L(\nu)]= \begin{cases}1 & \text { if } \gamma \in \mathcal{W}(\Lambda) . \nu \\ 0 & \text { otherwise. }\end{cases}
$$

(2) If $\Lambda$ is subgeneric, then we have for all $\gamma, \nu \in \Lambda$

$$
[\bar{\Delta}(\gamma): L(\nu)]= \begin{cases}1 & \text { if } \gamma \in\{\nu, \alpha \uparrow \nu\}, \\ 0 & \text { otherwise. }\end{cases}
$$


The above theorem confirms the Feigin-Frenkel conjecture in the respective cases. In AF09 we use it in order to describe the structure of the restricted category $\overline{\mathcal{O}}_{\Lambda}$ completely for subgeneric $\Lambda$. As for generic $\Lambda$ the structure of $\overline{\mathcal{O}}_{\Lambda}$ is easy to determine using the results of Feigin and Frenkel (see also Hay88, Ku89, Ma190, Mat96, we completed the first part of the Andersen-Jantzen-Soergel approach towards the description of the category $\mathcal{O}$ at the critical level.

\section{Affine KaC-Moody Algebras}

In this section we recall the fundamentals of the theory of affine Kac-Moody algebras. The main references are the textbooks Kac90 and MP95]. Our basic data is a finite-dimensional simple complex Lie algebra $\mathfrak{g}$. We denote by $k: \mathfrak{g} \times \mathfrak{g} \rightarrow$ $\mathbb{C}$ its Killing form.

2.1. The construction of $\widehat{\mathfrak{g}}$. From $\mathfrak{g}$ we construct the (untwisted) affine KacMoody algebra $\widehat{\mathfrak{g}}$ as follows. We first consider the loop algebra $\mathfrak{g} \otimes_{\mathbb{C}} \mathbb{C}\left[t, t^{-1}\right]$ for which the commutator is the $\mathbb{C}\left[t, t^{-1}\right]$-linear extension of the commutator of $\mathfrak{g}$. That means that we have $\left[x \otimes t^{n}, y \otimes t^{m}\right]=[x, y] \otimes t^{m+n}$ for $x, y \in \mathfrak{g}$ and $m, n \in \mathbb{Z}$. The loop algebra has an up to isomorphism unique non-split central extension $\widetilde{\mathfrak{g}}$ of rank one. As a vector space we have $\widetilde{\mathfrak{g}}=\mathfrak{g} \otimes_{\mathbb{C}} \mathbb{C}\left[t, t^{-1}\right] \oplus \mathbb{C} K$, and the Lie bracket is given by

$$
\begin{aligned}
{[K, \widetilde{\mathfrak{g}}] } & =0, \\
{\left[x \otimes t^{n}, y \otimes t^{m}\right] } & =[x, y] \otimes t^{m+n}+n \delta_{m,-n} k(x, y) K
\end{aligned}
$$

for $x, y \in \mathfrak{g}, n, m \in \mathbb{Z}$ (here $\delta_{a, b}$ denotes the Kronecker delta). In the last step of the construction we add the outer derivation operator $[D, \cdot]=t \frac{\partial}{\partial t}$ and get the affine Kac-Moody algebra $\widehat{\mathfrak{g}}:=\widetilde{\mathfrak{g}} \oplus \mathbb{C} D$ with the Lie bracket

$$
\begin{aligned}
{[K, \widehat{\mathfrak{g}}] } & =0, \\
{\left[D, x \otimes t^{n}\right] } & =n x \otimes t^{n}, \\
{\left[x \otimes t^{n}, y \otimes t^{m}\right] } & =[x, y] \otimes t^{m+n}+n \delta_{m,-n} k(x, y) K
\end{aligned}
$$

for $x, y \in \mathfrak{g}, n, m \in \mathbb{Z}$.

Let us fix a Borel subalgebra $\mathfrak{b} \subset \mathfrak{g}$ and a Cartan subalgebra $\mathfrak{h} \subset \mathfrak{b}$. Then the corresponding Borel subalgebra $\widehat{\mathfrak{b}}$ of $\widehat{\mathfrak{g}}$ is given by

$$
\widehat{\mathfrak{b}}:=\left(\mathfrak{g} \otimes_{\mathbb{C}} t \mathbb{C}[t]+\mathfrak{b} \otimes_{\mathbb{C}} \mathbb{C}[t]\right) \oplus \mathbb{C} K \oplus \mathbb{C} D
$$

and the Cartan subalgebra $\widehat{\mathfrak{h}} \subset \widehat{\mathfrak{b}}$ is given by

$$
\widehat{\mathfrak{h}}:=\mathfrak{h} \oplus \mathbb{C} K \oplus \mathbb{C} D .
$$

2.2. Affine roots. We denote by $V^{\star}$ the dual of a vector space $V$ and we write $\langle\cdot, \cdot\rangle: V^{\star} \times V \rightarrow \mathbb{C}$ for the canonical pairing. Let $R \subset \mathfrak{h}^{\star}$ be the set of roots of $\mathfrak{g}$ with respect to $\mathfrak{h}$. The projection $\widehat{\mathfrak{h}} \rightarrow \mathfrak{h}$ along the decomposition $\widehat{\mathfrak{h}}=\mathfrak{h} \oplus \mathbb{C} K \oplus \mathbb{C} D$ allows us to embed $\mathfrak{h}^{\star}$ inside $\widehat{\mathfrak{h}}^{\star}$. In particular, we can view any $\alpha \in R$ as an element 
in $\widehat{\mathfrak{h}}^{\star}$. Let us define $\delta, \Lambda_{0} \in \widehat{\mathfrak{h}}^{\star}$ by

$$
\begin{aligned}
\langle\delta, \mathfrak{h} \oplus \mathbb{C} K\rangle & =\{0\}, \\
\langle\delta, D\rangle & =1, \\
\left\langle\Lambda_{0}, \mathfrak{h} \oplus \mathbb{C} D\right\rangle & =\{0\}, \\
\left\langle\Lambda_{0}, K\right\rangle & =1 .
\end{aligned}
$$

Then we have $\widehat{\mathfrak{h}}^{\star}=\mathfrak{h}^{\star} \oplus \mathbb{C} \Lambda_{0} \oplus \mathbb{C} \delta$. The set $\widehat{R} \subset \widehat{\mathfrak{h}}^{\star}$ of roots of $\widehat{\mathfrak{g}}$ with respect to $\widehat{\mathfrak{h}}$ is

$$
\widehat{R}=\{\alpha+n \delta \mid \alpha \in R, n \in \mathbb{Z}\} \cup\{n \delta \mid n \in \mathbb{Z}, n \neq 0\} .
$$

For $\alpha \in R$ let us denote by $\mathfrak{g}_{\alpha} \subset \mathfrak{g}$ the corresponding root space. The root spaces of $\widehat{\mathfrak{g}}$ with respect to $\widehat{\mathfrak{h}}$ are

$$
\begin{aligned}
\widehat{\mathfrak{g}}_{\alpha+n \delta} & =\mathfrak{g}_{\alpha} \otimes t^{n} \quad \text { for } \alpha \in R, n \in \mathbb{Z}, \\
\widehat{\mathfrak{g}}_{n \delta} & =\mathfrak{h} \otimes t^{n} \quad \text { for } n \in \mathbb{Z}, n \neq 0 .
\end{aligned}
$$

The subsets

$$
\begin{aligned}
& \widehat{R}^{\mathrm{re}}:=\{\alpha+n \delta \mid \alpha \in R, n \in \mathbb{Z}\}, \\
& \widehat{R}^{\mathrm{im}}:=\{n \delta \mid n \in \mathbb{Z}, n \neq 0\}
\end{aligned}
$$

are called the sets of real roots and of imaginary roots, respectively.

Let $R^{+} \subset R$ be the set of roots of $\mathfrak{b}$ with respect to $\mathfrak{h}$. Then the set $\widehat{R}^{+}$of roots of $\widehat{\mathfrak{b}}$ with respect to $\widehat{\mathfrak{h}}$ is

$$
\widehat{R}^{+}=\{\alpha+n \delta \mid \alpha \in R, n \geq 1\} \cup R^{+} \cup\{n \delta \mid n \geq 1\} .
$$

Let $\Pi \subset R^{+}$be the set of simple roots and denote by $\gamma \in R^{+}$the highest root. Then the set of simple affine roots is

$$
\widehat{\Pi}=\Pi \cup\{-\gamma+\delta\} \subset \widehat{R}^{+} .
$$

For each real root $\alpha \in \widehat{R}^{\text {re }}$ the corresponding root space $\widehat{\mathfrak{g}}_{\alpha}$ is one-dimensional, and so is the commutator $\left[\widehat{\mathfrak{g}}_{\alpha}, \widehat{\mathfrak{g}}_{-\alpha}\right] \subset \widehat{\mathfrak{h}}$. The (affine) coroot $\alpha^{\vee}$ associated with $\alpha$ is the unique element in $\left[\widehat{\mathfrak{g}}_{\alpha}, \widehat{\mathfrak{g}}_{-\alpha}\right]$ on which $\alpha$ takes the value 2. Note that $\alpha^{\vee}$ is contained in $\mathfrak{h} \oplus \mathbb{C} K$, so we have $\left\langle\delta, \alpha^{\vee}\right\rangle=0$.

2.3. The Weyl groups. For $\alpha \in \widehat{R}^{\text {re }}$ we define the reflection $s_{\alpha}: \widehat{\mathfrak{h}}^{\star} \rightarrow \widehat{\mathfrak{h}}^{\star}$ by $s_{\alpha}(\lambda):=\lambda-\left\langle\lambda, \alpha^{\vee}\right\rangle \alpha$. We denote by $\widehat{\mathcal{W}} \subset \mathrm{GL}\left(\widehat{\mathfrak{h}}^{\star}\right)$ the affine Weyl group, i.e. the subgroup generated by the reflections $s_{\alpha}$ for $\alpha \in \widehat{R}^{+}$. The subgroup $\mathcal{W} \subset \widehat{\mathcal{W}}$ generated by the reflections $s_{\alpha}$ with $\alpha \in R$ leaves the subset $\mathfrak{h}^{\star} \subset \widehat{\mathfrak{h}}^{\star}$ stable and can be identified with the Weyl group of $\mathfrak{g}$.

Let $\rho \in \widehat{\mathfrak{h}}^{\star}$ be an element with the property $\left\langle\rho, \alpha^{\vee}\right\rangle=1$ for each simple affine root $\alpha$. Note that $\rho$ is only defined up to the addition of a multiple of $\delta$ (the span of the affine coroots is $\mathfrak{h} \oplus \mathbb{C} K$, and the simple coroots form a basis in this space). Yet all constructions in the following that use $\rho$ do not depend on this choice. So let us fix such an element $\rho$ once and for all.

The dot-action $\widehat{\mathcal{W}} \times \widehat{\mathfrak{h}}^{\star} \rightarrow \widehat{\mathfrak{h}}^{\star},(w, \lambda) \mapsto w \cdot \lambda$, of the affine Weyl group on $\widehat{\mathfrak{h}}^{\star}$ is obtained by shifting the linear action in such a way that $-\rho$ becomes a fixed point, i.e. it is given by

$$
w \cdot \lambda:=w(\lambda+\rho)-\rho
$$


for $w \in \widehat{\mathcal{W}}$ and $\lambda \in \widehat{\mathfrak{h}}^{\star}$. Note that since $\left\langle\delta, \alpha^{\vee}\right\rangle=0$ we have $s_{\alpha}(\delta)=\delta$ for all $\alpha \in \widehat{R}^{\text {re }}$. Hence $w(\delta)=\delta$ for all $w \in \widehat{\mathcal{W}}$ (so the dot-action is independent of the choice of $\rho$, as we claimed above).

2.4. The invariant bilinear form. Denote by $(\cdot, \cdot): \widehat{\mathfrak{g}} \times \widehat{\mathfrak{g}} \rightarrow \mathbb{C}$ the form given by

$$
\begin{aligned}
\left(x \otimes t^{n}, y \otimes t^{m}\right) & =\delta_{n,-m} k(x, y), \\
\left(K, \mathfrak{g} \otimes_{\mathbb{C}} \mathbb{C}\left[t, t^{-1}\right] \oplus \mathbb{C} K\right) & =\{0\}, \\
\left(D, \mathfrak{g} \otimes_{\mathbb{C}} \mathbb{C}\left[t, t^{-1}\right] \oplus \mathbb{C} D\right) & =\{0\}, \\
(K, D) & =1
\end{aligned}
$$

for $x, y \in \mathfrak{g}, m, n \in \mathbb{Z}$. It is non-degenerate, symmetric and invariant, i.e. it satisfies $([x, y], z)=(x,[y, z])$ for all $x, y, z \in \widehat{\mathfrak{g}}$. Moreover, it induces a non-degenerate bilinear form on the Cartan subalgebra $\widehat{\mathfrak{h}}$ and hence yields an isomorphism $\widehat{\mathfrak{h}} \breve{\rightarrow} \widehat{\mathfrak{h}}^{\star}$, which is the direct sum of the isomorphism $\mathfrak{h} \rightarrow \mathfrak{h}^{\star}$ given by the Killing form $k$ and the isomorphism $\mathbb{C} K \oplus \mathbb{C} D \rightarrow \mathbb{C} \Lambda_{0} \oplus \mathbb{C} \delta$ that maps $K$ to $\delta$ and $D$ to $\Lambda_{0}$. We get an induced symmetric non-degenerate form on the dual $\widehat{\mathfrak{h}}^{\star}$ that is given explicitly by

$$
\begin{aligned}
(\alpha, \beta) & =k(\alpha, \beta), \\
\left(\Lambda_{0}, \mathfrak{h}^{\star} \oplus \mathbb{C} \Lambda_{0}\right) & =\{0\}, \\
\left(\delta, \mathfrak{h}^{\star} \oplus \mathbb{C} \delta\right) & =\{0\}, \\
\left(\Lambda_{0}, \delta\right) & =1
\end{aligned}
$$

for $\alpha, \beta \in \mathfrak{h}^{\star}$ (here we also denote by $k$ the form on $\mathfrak{h}^{\star}$ that is induced by the Killing form). It is invariant under the linear action of the affine Weyl group, i.e. we have

$$
(w(\lambda), w(\mu))=(\lambda, \mu)
$$

for $w \in \widehat{\mathcal{W}}, \lambda, \mu \in \widehat{\mathfrak{h}}^{\star}$.

\section{The CATEgory $\mathcal{O}$ For an AFfine KaC-Moody algebra}

Having recalled the fundamental structural results for an affine Kac-Moody algebra we now turn to its representation theory. We restrict ourselves to representations in the affine category $\mathcal{O}$.

3.1. The category $\mathcal{O}$. Let $M$ be a $\widehat{\mathfrak{g}}$-module. Its weight space corresponding to $\lambda \in \widehat{\mathfrak{h}}^{\star}$ is

$$
M_{\lambda}:=\{m \in M \mid h \cdot m=\langle\lambda, h\rangle m \text { for all } h \in \widehat{\mathfrak{h}}\} .
$$

Any non-zero element $m \in M_{\lambda}$ is said to be of weight $\lambda$. We say that $M$ is a weight module if $M=\bigoplus_{\lambda \in \widehat{\mathfrak{h}}^{\star}} M_{\lambda}$. We say that $M$ is locally $\widehat{\mathfrak{b}}$-finite if all finitely generated $\widehat{\mathfrak{b}}$-submodules of $M$ are finite-dimensional. The affine category $\mathcal{O}$ is defined as the full subcategory of the category of $\widehat{\mathfrak{g}}$-modules that consists of all locally $\widehat{\mathfrak{b}}$-finite weight modules. 
3.2. Highest weight modules. Our choice of positive roots defines a partial order on $\widehat{\mathfrak{h}}^{\star}$ : we set $\nu \leqslant \nu^{\prime}$ if and only if $\nu^{\prime}-\nu \in \mathbb{Z}_{\geq 0} \widehat{R}^{+}$. A highest weight module of highest weight $\lambda \in \widehat{\mathfrak{h}}^{\star}$ is a $\widehat{\mathfrak{g}}$-module $M$ that contains a generator $v \neq 0$ of weight $\lambda$ such that $\widehat{\mathfrak{g}}_{\alpha} v=0$ for all $\alpha \in \widehat{R}^{+}$. Then $\lambda$ is indeed the highest weight of $M$, i.e. $M_{\mu} \neq 0$ implies $\mu \leqslant \lambda$. Each highest weight module is contained in $\mathcal{O}$.

For $\lambda \in \widehat{\mathfrak{h}}^{\star}$ denote by $\mathbb{C}_{\lambda}$ the one-dimensional $\widehat{\mathfrak{h}}$-module corresponding to $\lambda$. We extend the $\widehat{\mathfrak{h}}$-action to a $\widehat{\mathfrak{b}}$-action using the homomorphism $\widehat{\mathfrak{b}} \rightarrow \widehat{\mathfrak{h}}$ of Lie algebras that is left inverse to the inclusion $\widehat{\mathfrak{h}} \subset \widehat{\mathfrak{b}}$. That means that $\widehat{\mathfrak{g}}_{\alpha}$ acts trivially on $\mathbb{C}_{\lambda}$ for all $\alpha \in \widehat{R}^{+}$. The induced module

$$
\Delta(\lambda):=U(\widehat{\mathfrak{g}}) \otimes_{U(\widehat{\mathfrak{b}})} \mathbb{C}_{\lambda}
$$

is called the Verma module corresponding to $\lambda$. It contains a unique simple quotient $L(\lambda)$, and both $\Delta(\lambda)$ and $L(\lambda)$ are highest weight modules of highest weight $\lambda$.

Moreover, the modules $L(\lambda)$ for $\lambda \in \widehat{\mathfrak{h}}^{\star}$ form a full set of representatives of the simple isomorphism classes of $\mathcal{O}$, i.e. each simple object in $\mathcal{O}$ is isomorphic to $L(\lambda)$ for a unique $\lambda \in \widehat{\mathfrak{h}}^{\star}$.

3.3. Characters. Let $\mathbb{Z}\left[\widehat{\mathfrak{h}}^{\star}\right]=\bigoplus_{\lambda \in \widehat{\mathfrak{h}}^{\star}} \mathbb{Z} e^{\lambda}$ be the group algebra of the additive group $\widehat{\mathfrak{h}}^{\star}$. Let $\widehat{\mathbb{Z}\left[\widehat{\mathfrak{h}}^{\star}\right]} \subset \prod_{\lambda \in \widehat{\mathfrak{h}}^{\star}} \mathbb{Z} e^{\lambda}$ be the subgroup of elements $\left(c_{\lambda}\right)$ that have the property that there exists a finite set $\left\{\mu_{1}, \ldots, \mu_{n}\right\} \subset \widehat{\mathfrak{h}}^{\star}$ such that $c_{\lambda} \neq 0$ implies $\lambda \leq \mu_{i}$ for at least one $i$.

Let $\mathcal{O}^{f} \subset \mathcal{O}$ be the full subcategory of modules $M$ that have the property that the weight spaces $M_{\lambda}$ are finite-dimensional and such that there exist $\mu_{1}, \ldots, \mu_{n} \in \widehat{\mathfrak{h}}^{\star}$ such that $M_{\lambda} \neq 0$ implies $\lambda \leq \mu_{i}$ for at least one $i$. For each object $M$ of $\mathcal{O}^{f}$ we can then define its character as

$$
\operatorname{ch} M:=\sum_{\lambda \in \widehat{\mathfrak{h}}^{\star}}\left(\operatorname{dim}_{\mathbb{C}} M_{\lambda}\right) e^{\lambda} \in \widehat{\mathbb{Z}\left[\widehat{\mathfrak{h}}^{\star}\right]} .
$$

The character of a Verma module is easy to calculate. For each $\lambda \in \widehat{\mathfrak{h}}^{\star}$ we have

$$
\operatorname{ch} \Delta(\lambda)=e^{\lambda} \prod_{\alpha \in \widehat{R}^{+}}\left(1+e^{-\alpha}+e^{-2 \alpha}+\cdots\right)^{\operatorname{dim} \mathfrak{g}_{\alpha}} .
$$

(The above product is well-defined in $\widehat{\mathbb{Z}\left[\widehat{\mathfrak{h}}^{\star}\right]}$.) If $\lambda \in \widehat{\mathfrak{h}}^{\star}$ is non-critical (cf. Section 4), the character of $L(\lambda)$ is known (cf. [KT00, Fie06]). The principal aim of our research project is to calculate $\operatorname{ch} L(\lambda)$ for the critical highest weights $\lambda$.

3.4. Multiplicities. Suppose again that $M$ is an object in $\mathcal{O}^{f}$. Then there are well-defined numbers $a_{\nu} \in \mathbb{N}$ such that

$$
\operatorname{ch} M=\sum_{\nu \in \widehat{\mathfrak{h}}^{\star}} a_{\nu} \operatorname{ch} L(\nu)
$$

(cf. [DGK82]). Note that the sum on the right hand side is, in general, an infinite sum. We define the multiplicity of $L(\nu)$ in $M$ as

$$
[M: L(\nu)]:=a_{\nu}
$$


The matrix $[\Delta(\lambda): L(\mu)]$ is invertible, so the problem of calculating $\operatorname{ch} L(\mu)$ for all $\mu \in \widehat{\mathfrak{h}}^{\star}$ is equivalent to the calculation of the multiplicities $[\Delta(\lambda): L(\mu)]$ for all $\lambda, \mu \in \widehat{\mathfrak{h}}^{\star}$.

3.5. Block decomposition. Denote by " " the equivalence relation on $\widehat{\mathfrak{h}}^{\star}$ that is generated by $\lambda \sim \mu$ if $[\Delta(\lambda): L(\mu)] \neq 0$. For an equivalence class $\Lambda \in \widehat{\mathfrak{h}}^{\star} / \sim$ we define the full subcategory $\mathcal{O}_{\Lambda}$ of $\mathcal{O}$ that consists of all objects $M$ whose irreducible subquotients are isomorphic to $L(\lambda)$ for some $\lambda \in \Lambda$. We have the following decomposition result.

Theorem 3.1 ([DGK82, $\mathrm{RCW} 82])$. The functor

$$
\begin{aligned}
\prod_{\Lambda \in \widehat{\mathfrak{h}}^{\star} / \sim} \mathcal{O}_{\Lambda} & \rightarrow \mathcal{O}, \\
\left(M_{\Lambda}\right) & \mapsto \bigoplus_{\Lambda \in \widehat{\mathfrak{h}}^{\star} / \sim} M_{\Lambda}
\end{aligned}
$$

is an equivalence of categories.

3.6. The Kac-Kazhdan theorem. The following theorem gives an explicit description of the equivalence relation " " on $\widehat{\mathfrak{h}}^{\star}$. Let us denote by " $\preceq$ " the partial order on $\widehat{\mathfrak{h}}^{\star}$ generated by $\nu \preceq \lambda$ if there exist $n \in \mathbb{N}$ and $\beta \in \widehat{R}^{+}$such that $2(\lambda+\rho, \beta)=n(\beta, \beta)$ and $\nu=\lambda-n \beta$. In particular, $\nu \preceq \lambda$ implies $\nu \leqslant \lambda$, but the converse is not true.

Theorem 3.2 ([KK79]). We have $[\Delta(\lambda): L(\nu)] \neq 0$ if and only if $\nu \preceq \lambda$.

In particular, the equivalence relation " $\sim$ " is generated by the partial order relation "£”. For $\Lambda \in \widehat{\mathfrak{h}}^{\star} / \sim$ set

$$
\begin{aligned}
\widehat{R}(\Lambda) & :=\{\alpha \in \widehat{R} \mid 2(\lambda+\rho, \alpha) \in \mathbb{Z}(\alpha, \alpha) \text { for some } \lambda \in \Lambda\} \\
& =\{\alpha \in \widehat{R} \mid 2(\lambda+\rho, \alpha) \in \mathbb{Z}(\alpha, \alpha) \text { for all } \lambda \in \Lambda\}, \\
\widehat{\mathcal{W}}(\Lambda) & :=\left\langle s_{\alpha} \mid \alpha \in \widehat{R}(\Lambda) \cap \widehat{R}^{\mathrm{re}}\right\rangle \subset \widehat{\mathcal{W}} .
\end{aligned}
$$

If $\Lambda$ is non-critical, i.e. if $\widehat{R}(\Lambda) \subset \widehat{R}^{\mathrm{re}}$, then $\Lambda=\widehat{\mathcal{W}}(\Lambda) . \lambda$ for each $\lambda \in \Lambda$. In this case, the structure of the block $\mathcal{O}_{\Lambda}$ can be completely described in terms of the group $\widehat{\mathcal{W}}(\Lambda)$ (which turns out to be a Coxeter group) and the singularity of its orbit $\Lambda$; cf. [Fie06].

3.7. A duality on $\mathcal{O}^{f}$. We will later need the following duality functor. For convenience we only define it on the full subcategory $\mathcal{O}^{f}$ of $\mathcal{O}$ that we defined earlier. All the modules that we encounter in this article belong to $\mathcal{O}^{f}$.

For $M \in \mathcal{O}^{f}$ we set $M^{\star}:=\bigoplus_{\lambda \in \widehat{\mathfrak{h}}^{\star}} \operatorname{Hom}_{\mathbb{C}}\left(M_{\lambda}, \mathbb{C}\right)$. We endow $M^{\star}$ with the action of $\widehat{\mathfrak{g}}$ given by

$$
(x . \phi)(m)=\phi(-\omega(x) \cdot m),
$$

for $x \in \widehat{\mathfrak{g}}, \phi \in M^{\star}$ and $m \in M$, where $\omega: \widehat{\mathfrak{g}} \rightarrow \widehat{\mathfrak{g}}$ is the Chevalley-involution, i.e. the involution induced on $\widehat{\mathfrak{g}}$ by the root system automorphism that sends $\alpha \in \widehat{R}$ to $-\alpha \in \widehat{R}$ (cf. Kac90, Section 1.3]). Then $M^{\star} \in \mathcal{O}^{f}$ and we indeed get a duality functor on $\mathcal{O}^{f}$. It is exact and maps irreducible modules to irreducible modules. A quick look at characters shows the following. 
Lemma 3.3. For each $\lambda \in \widehat{\mathfrak{h}}^{\star}$ we have $L(\lambda)^{\star}=L(\lambda)$.

For each $\lambda \in \widehat{\mathfrak{h}}^{\star}$ we denote by

$$
\nabla(\lambda):=\Delta(\lambda)^{\star}
$$

the dual of the Verma module with highest weight $\lambda$. By the above lemma and the exactness of the duality, $\nabla(\lambda)$ and $\Delta(\lambda)$ have the same Jordan-Hölder multiplicities, and $\nabla(\lambda)$ has a simple socle which is isomorphic to $L(\lambda)$.

\section{The CRITICAL HYPERPLANE}

In this section we recall the notion of a critical weight for the affine Kac-Moody algebra $\widehat{\mathfrak{g}}$. We introduce a shift functor $T$ on each of the critical blocks and study the corresponding graded center $\mathcal{A}=\bigoplus_{n \in \mathbb{Z}} \mathcal{A}_{n}$. For a critical weight $\lambda \in \widehat{\mathfrak{h}}^{\star}$ we define the restricted Verma module $\bar{\Delta}(\lambda)$ as the quotient of $\Delta(\lambda)$ by the ideal of $\mathcal{A}$ generated by $\bigoplus_{n \neq 0} \mathcal{A}_{n}$. We state some fundamental properties of these modules in Theorem 4.7. The proof of this theorem is due to Feigin and Frenkel. Then we recall the Feigin-Frenkel conjecture on the Jordan-Hölder multiplicities for restricted Verma modules and, finally, state the main result of this article in Theorem 4.9.

4.1. A shift functor. The defining relations of $\widehat{\mathfrak{g}}$ show that the derived Lie algebra of $\widehat{\mathfrak{g}}$ coincides with the central extension $\widetilde{\mathfrak{g}}$ of the loop algebra, i.e.

$$
[\widehat{\mathfrak{g}}, \widehat{\mathfrak{g}}]=\mathfrak{g} \otimes \mathbb{C}\left[t, t^{-1}\right] \oplus \mathbb{C} K
$$

Hence $[\widehat{\mathfrak{g}}, \widehat{\mathfrak{g}}]$ is of codimension one in $\widehat{\mathfrak{g}}$, so the quotient $\widehat{\mathfrak{g}} /[\widehat{\mathfrak{g}}, \widehat{\mathfrak{g}}]$ is a one-dimensional Lie algebra. Each character of $\widehat{\mathfrak{g}} /[\widehat{\mathfrak{g}}, \widehat{\mathfrak{g}}]$ gives rise to a one-dimensional module of $\widehat{\mathfrak{g}}$. In this way we get the simple modules $L(\zeta \delta)$ for $\zeta \in \mathbb{C}$. We have $L(\zeta \delta) \otimes L(\xi \delta) \cong$ $L((\zeta+\xi) \delta)$ for $\zeta, \xi \in \mathbb{C}$.

Let us define the shift functor

$$
\begin{aligned}
T: \widehat{\mathfrak{g}}-\bmod & \rightarrow \widehat{\mathfrak{g}}-\bmod , \\
M & \mapsto M \otimes_{\mathbb{C}} L(\delta) .
\end{aligned}
$$

The action of $\widehat{\mathfrak{g}}$ on the tensor product is the usual one: $X .(m \otimes l)=X . m \otimes l+m \otimes X . l$ for $X \in \widehat{\mathfrak{g}}, m \in M$ and $l \in L(\delta)$. The functor $T$ is exact and preserves the categories $\mathcal{O}^{f}$ and $\mathcal{O}$. Clearly, it is an equivalence on these categories with inverse $T^{-1}: M \mapsto$ $M \otimes_{\mathbb{C}} L(-\delta)$. For $n \in \mathbb{Z}$ we denote by $T^{n}: \mathcal{O} \rightarrow \mathcal{O}$ the $|n|$-fold composition of $T$ or of $T^{-1}$. It is given by the tensor product with the one-dimensional module $L(n \delta)$.

The following lemma is easy to prove (for part (3) use the fact that $L(\delta)^{\star} \cong L(\delta)$ and $\left.\left(M \otimes_{\mathbb{C}} N\right)^{\star}=M^{\star} \otimes_{\mathbb{C}} N^{\star}\right)$.

Lemma 4.1. For each $\lambda \in \widehat{\mathfrak{h}}^{\star}$ we have

(1) $T \Delta(\lambda) \cong \Delta(\lambda+\delta)$,

(2) $T L(\lambda) \cong L(\lambda+\delta)$,

(3) on $\mathcal{O}^{f}$ the functor $T$ commutes with the duality, i.e. there is a natural equivalence $T \circ(\cdot)^{\star} \cong(\cdot)^{\star} \circ T$ of functors.

Let us denote by $\lambda \mapsto \bar{\lambda}$ the linear map $\widehat{\mathfrak{h}}^{\star} \rightarrow \mathfrak{h}^{\star}$ that is dual to the inclusion $\mathfrak{h} \subset \widehat{\mathfrak{h}}$. For a subset $\Lambda$ of $\widehat{\mathfrak{h}}^{\star}$ we denote by $\bar{\Lambda}$ its image in $\mathfrak{h}^{\star}$. Now we come to the definition of critical equivalence classes, critical weights and critical blocks. 
Lemma 4.2. For an equivalence class $\Lambda \in \widehat{\mathfrak{h}}^{\star} / \sim$ the following are equivalent:

(1) The functor $T$ maps $\mathcal{O}_{\Lambda}$ into itself.

(2) We have $\lambda+\delta \sim \lambda$ for all $\lambda \in \Lambda$.

(3) We have $\mathbb{Z} \delta \backslash\{0\}=\widehat{R}^{\mathrm{im}} \subset \widehat{R}(\Lambda)$.

(4) For all $\lambda \in \Lambda$, the restriction of $\lambda$ to the central line $\mathbb{C} K \subset \widehat{\mathfrak{h}}$ coincides with the restriction of $-\rho$, i.e. we have $\langle\lambda, K\rangle=\langle-\rho, K\rangle$.

(5) The induced dot-action of the affine Weyl group $\widehat{\mathcal{W}}$ on $\bar{\Lambda}$ factors over an action of the finite Weyl group.

Remark 4.3. Note that $w \cdot(\lambda+\xi \delta)=w \cdot \lambda+\xi w(\delta)=w \cdot \lambda+\xi \delta$, so $w \cdot \lambda \equiv w \cdot(\lambda+\xi \delta)$ $\bmod \delta$ for all $\lambda$, so the dot-action indeed induces an action of $\widehat{\mathcal{W}}$ on the quotient $\widehat{\mathfrak{h}}^{\star} / \mathbb{C} \delta$ and the statement (5) above makes sense.

If the above conditions on $\Lambda$ are satisfied, we say that $\Lambda$ is a critical equivalence class. In this case we call each element $\lambda$ of $\Lambda$ a critical weight, or of critical level. We let $\widehat{\mathfrak{h}}_{\text {crit }}^{\star}$ be the set of weights of critical level, i.e. the union of the critical equivalence classes. Condition (4) above shows that $\widehat{\mathfrak{h}}_{\text {crit }}^{\star}$ is an affine hyperplane in $\widehat{\mathfrak{h}}^{\star}$. It is called the critical hyperplane.

Proof. From the definition of the blocks, the exactness of $T$ and Lemma 4.1 we deduce the equivalence of (1) and (2).

Note that $(\alpha+n \delta)^{\vee}=\alpha^{\vee}+n K^{\prime}$, where $K^{\prime}$ is a non-zero multiple of $K$. Hence, $\lambda \in \widehat{\mathfrak{h}}^{\star}$ has the property that $s_{\alpha+n \delta} . \lambda \equiv s_{\alpha+m \delta} . \lambda \bmod \delta$ for all $m, n \in \mathbb{Z}$ if and only if $\langle\lambda+\rho, K\rangle=0$. Hence (4) and (5) are equivalent.

As $(\delta, \delta)=0$ we have $\mathbb{Z} \delta \backslash\{0\} \subset \widehat{R}(\Lambda)$ if and only if $(\lambda+\rho, \delta)=0$ for all $\lambda \in \Lambda$. The latter equation is equivalent to $\langle\lambda+\rho, K\rangle=0$. Hence (3) and (4) are equivalent.

Clearly, (3) implies (2). On the other hand, suppose that (2) holds, but (3) does not hold. Then $\widehat{R}^{\mathrm{im}} \cap \widehat{R}(\Lambda)=\emptyset$, and $\Lambda$ is an orbit of $\widehat{\mathcal{W}}(\Lambda)$ under the dot-action. So $\lambda, \lambda+\delta \in \Lambda$ implies that $\lambda+\delta$ and $\lambda$ are contained in the same $\widehat{\mathcal{W}}$-orbit. The invariance of the bilinear form then yields $(\lambda+\delta+\rho, \lambda+\delta+\rho)=(\lambda+\rho, \lambda+\rho)$ which implies, as $(\delta, \delta)=0$, that $(\lambda+\rho, \delta)=0$, hence $\langle\lambda+\rho, K\rangle=0$, hence (4), which is equivalent to (3). Hence we have a contradiction. So (2) implies (3).

4.2. The action of $\widehat{\mathcal{W}}$ in the critical hyperplane. Let us fix a critical equivalence class $\Lambda \subset \widehat{\mathfrak{h}}_{\text {crit }}^{\star}$. Then we have $\delta \in \widehat{R}(\Lambda)$, so $(\lambda+\rho, \delta) \in \mathbb{Z}(\delta, \delta)=0$ for some (all) $\lambda \in \Lambda$. Hence, if $\alpha \in \widehat{R}(\Lambda)$, then either $\alpha=-\delta$ or $\alpha+\delta \in \widehat{R}(\Lambda)$. If we set $R(\Lambda):=R \cap \widehat{R}(\Lambda)$, then

$$
\widehat{R}(\Lambda)=\{\alpha+n \delta \mid \alpha \in R(\Lambda), n \in \mathbb{Z}\} \cup\{n \delta \mid n \in \mathbb{Z}, n \neq 0\} .
$$

Moreover, we have $s_{\alpha+n \delta} \in \widehat{\mathcal{W}}(\Lambda)$ if and only if $s_{\alpha} \in \widehat{\mathcal{W}}(\Lambda)$. We set $\mathcal{W}(\Lambda)=$ $\widehat{\mathcal{W}}(\Lambda) \cap \mathcal{W}$. Then $\widehat{\mathcal{W}}(\Lambda)$ is the affinization of $\mathcal{W}(\Lambda)$.

Let $\alpha \in R(\Lambda)$. We now define a bijection $\alpha \uparrow:: \Lambda \rightarrow \Lambda$, following [AJS94. For $\lambda \in \Lambda$ let $\alpha \uparrow \lambda$ be the minimal element in $\left\{s_{\alpha, n} . \lambda \mid n \in \mathbb{Z}, s_{\alpha, n} . \lambda \geq \lambda\right\}$. Note that $\alpha \uparrow \lambda=\lambda$ if $\lambda$ is contained in the reflection hyperplane corresponding to some $s_{\alpha, n}$. We denote by $\alpha \downarrow \cdot: \Lambda \rightarrow \Lambda$ the inverse map. 
Definition 4.4. We say that

(1) $\Lambda$ is generic if $\bar{\Lambda}$ contains only one element,

(2) $\Lambda$ is subgeneric if $\bar{\Lambda}$ contains exactly two elements,

(3) $\Lambda$ is regular if for some (all) $\nu$ in $\Lambda$ we have that $w \cdot \nu=\nu$ implies $w=e$ for all $w \in \mathcal{W}$.

Note that part (3) of the above definition refers only to the action of the finite Weyl group. If $\Lambda$ is subgeneric, then there is a unique finite positive root $\alpha$ such that $\bar{\Lambda}=\left\{\bar{\lambda}, s_{\alpha} \cdot \bar{\lambda}\right\}$. Set $R(\Lambda)^{+}:=R(\Lambda) \cap R^{+}$.

Definition 4.5. Let $\nu \in \Lambda$. We say that

(1) $\nu$ is dominant if $\left\langle\nu+\rho, \alpha^{\vee}\right\rangle \geq 0$ for all $\alpha \in R(\Lambda)^{+}$.

(2) $\nu$ is anti-dominant if $\left\langle\nu+\rho, \alpha^{\vee}\right\rangle \leq 0$ for all $\alpha \in R(\Lambda)^{+}$.

4.3. The graded center of a critical block. Since $\Lambda$ is supposed to be critical, we can consider the functor $T$ as an auto-equivalence on the block $\mathcal{O}_{\Lambda}$.

Let $n \in \mathbb{Z}$ and let $z$ be a natural transformation from the functor $T^{n}$ on $\mathcal{O}_{\Lambda}$ to the identity functor id on $\mathcal{O}_{\Lambda}$. Note that $z$ associates with any $M \in \mathcal{O}_{\Lambda}$ a homomorphism $z^{M}: T^{n} M \rightarrow M$ in such a way that for any homomorphism $f: M \rightarrow$ $N$ in $\mathcal{O}_{\Lambda}$ the diagram

commutes.

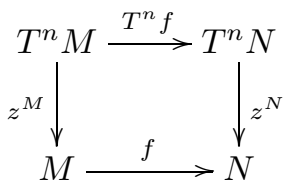

Denote by $\mathcal{A}_{n}=\mathcal{A}_{n}(\Lambda)$ the complex vector space of all natural transformations $z$ as above such that $z^{T^{l} M}=T^{l} z^{M}: T^{n+l} M \rightarrow T^{l} M$ for all $M \in \mathcal{O}_{\Lambda}$ and $l \in \mathbb{Z}$. There is a bilinear map

$$
\begin{aligned}
\mathcal{A}_{n} \times \mathcal{A}_{m} & \rightarrow \mathcal{A}_{m+n}, \\
\left(z_{1}, z_{2}\right) & \mapsto\left(M \mapsto z_{1}^{M} \circ\left(T^{n} z_{2}^{M}\right)\right)
\end{aligned}
$$

that makes $\mathcal{A}=\mathcal{A}(\Lambda):=\bigoplus_{n \in \mathbb{Z}} \mathcal{A}_{n}$ into a graded $\mathbb{C}$-algebra. It is associative, commutative and unital.

4.4. Restricted Verma modules. Let $\lambda \in \Lambda$ and $n \in \mathbb{Z}$. Each $z \in \mathcal{A}_{n}$ defines a homomorphism

$$
z^{\Delta(\lambda)}: T^{n} \Delta(\lambda) \cong \Delta(\lambda+n \delta) \rightarrow \Delta(\lambda) .
$$

Each such homomorphism is zero if $n>0$. Define $\Delta(\lambda)^{-} \subset \Delta(\lambda)$ as the submodule generated by the images of all the homomorphisms $z^{\Delta(\lambda)}$ for $z \in \mathcal{A}_{n}$ and $n<0$.

Definition 4.6. The quotient

$$
\bar{\Delta}(\lambda):=\Delta(\lambda) / \Delta(\lambda)^{-}
$$

is called the restricted Verma module of highest weight $\lambda$.

Consider the formal power series

$$
\prod_{j \geq 1}\left(1-q^{j}\right)^{-\operatorname{rank} \mathfrak{g}}=\prod_{j \geq 1}\left(1+q^{j}+q^{2 j}+\cdots\right)^{\text {rank } \mathfrak{g}},
$$

and let us define for $n \in \mathbb{Z}$ the number $p(n) \in \mathbb{N}$ as the coefficient of $q^{n}$ in the above series. 
We denote by $\widehat{\mathfrak{n}}_{+}:=\bigoplus_{\alpha \in \widehat{R}^{+}} \widehat{\mathfrak{g}}_{\alpha}$ the subalgebra of $\widehat{\mathfrak{g}}$ corresponding to the positive affine roots. For a $\widehat{\mathfrak{g}}$-module $M$ we denote by $M^{\widehat{\mathfrak{n}}_{+}}$the set of $\widehat{\mathfrak{n}}_{+}$-invariant vectors. It is an $\widehat{\mathfrak{h}}$-submodule of $M$, hence we can also define its weight spaces $M_{\nu}^{\widehat{\mathfrak{n}}_{+}}$for $\nu \in \widehat{\mathfrak{h}}^{\star}$.

The following theorem lists the most important properties of the restricted Verma modules. The proofs of the following statements (2), (3) and (4), as well as the main step in the proof of (1), are due to Feigin and Frenkel. We recall the main arguments in Section 5 .

Theorem 4.7. Let $\lambda \in \widehat{\mathfrak{h}}_{\text {crit }}^{\star}$.

(1) The map $\mathcal{A}_{n} \rightarrow \operatorname{Hom}\left(T^{n} \Delta(\lambda), \Delta(\lambda)\right)$ is surjective for all $n \in \mathbb{Z}$.

(2) We have $\operatorname{dim} \operatorname{Hom}\left(T^{n} \Delta(\lambda), \Delta(\lambda)\right)=p(-n)$ for all $n \in \mathbb{Z}$.

(3) We have

$$
\operatorname{ch} \bar{\Delta}(\lambda)=e^{\lambda} \prod_{\alpha \in \widehat{R}^{+} \cap \widehat{R}^{\mathrm{re}}}\left(1+e^{-\alpha}+e^{-2 \alpha}+\ldots\right) .
$$

(4) We have $\bar{\Delta}(\lambda)_{\lambda-n \delta}^{\widehat{\mathfrak{n}}_{+}}=\{0\}$ for $n \neq 0$.

4.5. A conjecture. The character of $L(\lambda)$ for a critical highest weight $\lambda$ is not yet known in general. But we have a formula for the characters of the restricted Verma modules and the simple characters can be calculated once the Jordan-Hölder multiplicities $[\Delta(\lambda): L(\mu)]$ for critical weights $\lambda$ and $\mu$ are determined. In the following we state a conjecture that gives a formula for these multiplicities in terms of periodic Kazhdan-Lusztig polynomials. It is due to Feigin and Frenkel.

Let $\Lambda$ be a critical equivalence class. Then $\widehat{\mathcal{W}}(\Lambda)$ is the affinization of the finite Weyl group $\mathcal{W}(\Lambda)$. So we can think of $\widehat{\mathcal{W}}(\Lambda)$ as a group of affine transformations on a vector space. The connected complements of the affine reflection hyperplanes are called alcoves, and the set of alcoves is a principal homogeneous set for the action of $\widehat{\mathcal{W}}(\Lambda)$. Let $A_{e}$ be the unique alcove in the dominant Weyl chamber that contains the origin in its closure. Then the map $w \mapsto A_{w}:=w\left(A_{e}\right)$ gives a bijection between $\widehat{\mathcal{W}}(\Lambda)$ and the set of alcoves. In Lus80. Lusztig defined for alcoves $A$ and $B$ a periodic polynomial $p_{A, B} \in \mathbb{Z}[v]$ (we use the normalization and notation of Soergel; cf. [Soe97] and [Fie07]). Denote by $w_{\Lambda} \in \mathcal{W}(\Lambda)$ the longest element.

Conjecture 4.8. Let $\Lambda$ be a critical equivalence class.

(1) The restricted linkage principle: For $\lambda, \mu \in \Lambda$ we have that $[\bar{\Delta}(\lambda): L(\mu)] \neq$ 0 implies $\mu \in \widehat{\mathcal{W}}(\Lambda) \cdot \lambda$.

(2) The restricted Verma multiplicities: Let $\lambda \in \Lambda$ be regular and dominant and $w \in \widehat{\mathcal{W}}(\Lambda)$. Suppose that for all $x, x^{\prime} \in \widehat{\mathcal{W}}(\Lambda)$ with $p_{A_{w_{\Lambda}}, A_{w_{\Lambda} w}}(1) \neq 0$ and $p_{A_{w_{\Lambda} x^{\prime}}, A_{w_{\Lambda}}}(1) \neq 0$ and $x \neq x^{\prime}$ we have $x . \lambda \neq x^{\prime} . \lambda$. Then

$$
[\bar{\Delta}(w \cdot \lambda): L(x \cdot \lambda)]=p_{A_{w_{\Lambda} w}, A_{w_{\Lambda} x}}(1)
$$

for all $x \in \widehat{\mathcal{W}}(\Lambda)$.

This conjecture is closely related to an anticipated relation between representations of a small quantum group, the topology of semi-infinite flag manifolds and the restricted critical level representations of an affine Kac-Moody algebra; cf. Lus91. We prove part (1) of the above conjecture in AF09. 
4.6. The main result. In the following theorem we summarize the main results of this article.

Theorem 4.9. Suppose that $\Lambda \subset \widehat{\mathfrak{h}}^{\star}$ is a critical equivalence class.

(1) If $\nu \in \Lambda$ is anti-dominant, then for all $w \in \mathcal{W}(\Lambda)$ and $n \geq 0$ we have

$$
[\Delta(w . \nu): L(\nu-n \delta)]=p(n) .
$$

(2) If $\Lambda$ is subgeneric and $\nu \in \Lambda$ is dominant, then we have for all $n \geq 0$

$$
[\Delta(\nu): L(\nu-n \delta)]=[\Delta(\alpha \uparrow \nu): L(\nu-n \delta)]=p(n),
$$

where $\alpha$ is the unique positive finite root with $\bar{\Lambda}=\left\{\bar{\lambda}, s_{\alpha} \cdot \bar{\lambda}\right\}$.

Let us restate the above results in terms of restricted Verma modules.

Corollary 4.10. Let $\Lambda \subset \widehat{\mathfrak{h}}^{\star}$ be a critical equivalence class.

(1) Suppose that $\nu \in \Lambda$ is anti-dominant. Then for any $\gamma \in \Lambda$ we have

$$
[\bar{\Delta}(\gamma): L(\nu)]=\left\{\begin{array}{l}
1 \text { if } \gamma \in \mathcal{W}(\Lambda) . \nu, \\
0 \text { else. }
\end{array}\right.
$$

In particular, Conjecture 4.8 holds for the anti-dominant multiplicities.

(2) If $\Lambda$ is subgeneric, then we have for all $\gamma, \nu \in \Lambda$

$$
[\bar{\Delta}(\gamma): L(\nu)]= \begin{cases}1 & \text { if } \nu \in\{\gamma, \alpha \downarrow \gamma\}, \\ 0 & \text { else, }\end{cases}
$$

where $\alpha \in R^{+}$is such that $\bar{\Lambda}=\left\{\bar{\lambda}, s_{\alpha} \cdot \bar{\lambda}\right\}$. In particular, Conjecture 4.8 holds in the subgeneric cases.

In the following section we recall the results of Feigin and Frenkel on the center at the critical level and deduce Theorem 4.7. In Section 6 we study the structure of projective objects in a critical block $\mathcal{O}_{\Lambda}$. In particular, we provide some results on the action of the graded center $\mathcal{A}$ on a projective object. In Section 7 we introduce the BRST-cohomology functor and recall the main Theorem of Ara07. In Section 8 we use the results of Sections 5 , 6 and 7 to give a proof of Theorem 4.9.

\section{The Feigin-Frenkel Center}

In this section we recall the fundamental results on the Feigin-Frenkel center FF92 at the critical level. The main references are the textbooks [FBZ04 and Fre07.

\subsection{The universal affine vertex algebra at the critical level. Set}

$$
V^{\text {crit }}(\mathfrak{g}):=U(\widehat{\mathfrak{g}}) \otimes_{U\left(\mathfrak{g} \otimes_{\mathbb{C}} \mathbb{C}[t] \oplus \mathbb{C} K \oplus \mathbb{C} D\right)} \mathbb{C}_{\text {crit }},
$$

where $\mathbb{C}_{\text {crit }}$ is the one-dimensional representation of $\mathfrak{g} \otimes_{\mathbb{C}} \mathbb{C}[t] \oplus \mathbb{C} K \oplus \mathbb{C} D$ on which $\mathfrak{g} \otimes_{\mathbb{C}} \mathbb{C}[t] \oplus \mathbb{C} D$ acts trivially and $K$ acts as multiplication by the critical value $\langle-\rho, K\rangle$. The space $V^{\text {crit }}(\mathfrak{g})$ has a natural structure of a vertex algebra and is called the universal affine vertex algebra associated with $\mathfrak{g}$ at the critical level (see e.g., Kac98, §4.9]). Let

$$
\begin{aligned}
Y(?, z): V^{\text {crit }}(\mathfrak{g}) & \rightarrow \operatorname{End}_{\mathbb{C}} V^{\text {crit }}(\mathfrak{g})\left[\left[z, z^{-1}\right]\right], \\
a & \mapsto a(z)=\sum_{n \in \mathbb{Z}} a_{(n)} z^{-n-1}
\end{aligned}
$$


be the state-field correspondence. The map $Y(?, z)$ is uniquely determined by the condition

$$
Y\left(\left(x \otimes t^{-1}\right) \mathbf{1}, z\right)=\sum_{n \in \mathbb{Z}}\left(x \otimes t^{n}\right) z^{-n-1} \quad \text { for } x \in \mathfrak{g},
$$

where 1 is the vacuum vector $1 \otimes 1$.

The vertex algebra $V^{\text {crit }}(\mathfrak{g})$ is graded by the Hamiltonian $-D$. If $a \in V^{\text {crit }}(\mathfrak{g})$ is an eigenvector of $-D$, its eigenvalue is called the conformal weight and is denoted by $\Delta_{a}$. We denote by $\partial$ the translation operator. It satisfies

$$
Y(\partial a, z)=[\partial, Y(a, z)]=\frac{d}{d z} Y(a, z) .
$$

5.2. The Feigin-Frenkel center. Let $\mathfrak{z}(\widehat{\mathfrak{g}})$ be the center of the vertex algebra $V^{\text {crit }}(\mathfrak{g})$ :

$$
\mathfrak{z}(\widehat{\mathfrak{g}})=\left\{a \in V^{\text {crit }}(\mathfrak{g}) \mid\left[a_{(m)}, v_{(n)}\right]=0 \text { for all } v \in V^{\text {crit }}(\mathfrak{g}), m, n \in \mathbb{Z}\right\} .
$$

One has

$$
\begin{aligned}
\mathfrak{z}(\widehat{\mathfrak{g}}) & =\left\{a \in V^{\text {crit }}(\mathfrak{g}) \mid v_{(n)} a=0 \text { for all } v \in V^{\text {crit }}(\mathfrak{g}), n \geq 0\right\} \\
& =V^{\text {crit }}(\mathfrak{g})^{G[[t]]},
\end{aligned}
$$

where $G$ is the adjoint group of $\mathfrak{g}$ and $G[[t]]$ is the $\mathbb{C}[[t]]$-points of $G$.

Let $\left\{U_{p}\left(\mathfrak{g} \otimes_{\mathbb{C}} \mathbb{C}\left[t^{-1}\right] t^{-1}\right)\right\}$ be the standard filtration of $U\left(\mathfrak{g} \otimes_{\mathbb{C}} \mathbb{C}\left[t^{-1}\right] t^{-1}\right)$ and set

$$
F_{p} V^{\text {crit }}(\mathfrak{g}):=U_{p}\left(\mathfrak{g} \otimes_{\mathbb{C}} \mathbb{C}\left[t^{-1}\right] t^{-1}\right) \cdot \mathbf{1} \subset V^{\text {crit }}(\mathfrak{g}) .
$$

This defines a filtration of a vertex algebra. Let gr $V^{\text {crit }}(\mathfrak{g})$ be the associated graded vertex algebra: gr $V^{\text {crit }}(\mathfrak{g})=\bigoplus_{p} F_{p} V^{\text {crit }}(\mathfrak{g}) / F_{p-1} V^{\text {crit }}(\mathfrak{g})$. It is a commutative vertex algebra and one has

$$
\operatorname{gr} V^{\text {crit }}(\mathfrak{g}) \cong S\left(\mathfrak{g}\left[t^{-1}\right] t^{-1}\right) \cong \mathbb{C}\left[\mathfrak{g}_{\infty}\right]
$$

as differential ring $₫ 1$ and $G[[t]]$-modules, where $\mathfrak{g}_{\infty}$ is the infinite jet scheme of $\mathfrak{g}$ (cf. EF01]) and $\mathfrak{g}$ is identified with $\mathfrak{g}^{\star}$. Below we shall identify gr $V^{\text {crit }}(\mathfrak{g})$ with $\mathbb{C}\left[\mathfrak{g}_{\infty}\right]$. The natural projection $\mathfrak{g}_{\infty} \rightarrow \mathfrak{g}$ gives the embedding $\mathbb{C}[\mathfrak{g}] \hookrightarrow \mathbb{C}\left[\mathfrak{g}_{\infty}\right]$.

Let $\left\{F_{p \mathfrak{z}}(\widehat{\mathfrak{g}})\right\}$ be the induced filtration of $\mathfrak{z}(\widehat{\mathfrak{g}})$, and let $\operatorname{gr} \mathfrak{z}(\widehat{\mathfrak{g}})$ be the associated graded vertex algebra. Certainly, the image of the natural embedding gr $\mathfrak{z}(\widehat{\mathfrak{g}}) \hookrightarrow$ gr $V^{\text {crit }}(\mathfrak{g})=\mathbb{C}\left[\mathfrak{g}_{\infty}\right]$ is contained in $\mathbb{C}\left[\mathfrak{g}_{\infty}\right]^{G[t]]}$.

Let $\bar{p}^{(1)}, \ldots, \bar{p}^{(\ell)}$, where $\ell=\operatorname{rank} \mathfrak{g}$, be homogeneous generators of the ring $\mathbb{C}[\mathfrak{g}]^{G} \subset \mathbb{C}\left[\mathfrak{g}_{\infty}\right]^{G[[t]]}$. The elements $\bar{p}_{(-j-1)}^{(i)}:=(\partial)^{j} \bar{p}^{(i)} / j$ ! are also $G[[t]]$-invariant for all $j \geq 0$.

According to [BD96] (see also [EF01]), one has

$$
\mathbb{C}\left[\mathfrak{g}_{\infty}\right]^{G[[t]]}=\mathbb{C}\left[\bar{p}_{(-j-1)}^{(1)}, \ldots, \bar{p}_{(-j-1)}^{(\ell)}\right]_{j \geq 0} .
$$

Theorem 5.1 ([FF92]; see also [Fre07]). The embedding

$$
\operatorname{gr} \mathfrak{z}(\widehat{\mathfrak{g}}) \rightarrow \mathbb{C}\left[\mathfrak{g}_{\infty}\right]^{G[t]]]}
$$

is an isomorphism.

\footnotetext{
${ }^{1}$ A commutative vertex algebra $V$ is naturally a commutative ring with a derivation by the multiplication $(a, b) \mapsto a_{(-1)} b$ and the derivation $\partial a_{(-n)}=n a_{(-n-1)}$.
} 
By Theorem 5.1 there exist homogeneous generators $p^{(1)}, \ldots, p^{(\ell)}$ of $\mathfrak{z}(\widehat{\mathfrak{g}})$ whose symbols are $\bar{p}^{(1)}, \ldots, \bar{p}^{(\ell)}$. Let $d_{i}=\operatorname{deg} \bar{p}^{(i)}-1$, so that $d_{1}, \ldots, d_{\ell}$ are the exponents of $\mathfrak{g}$. The conformal weight of $p^{(i)}$ is by definition $d_{i}+1$. We write

$$
Y\left(p^{(i)}, z\right)=p^{(i)}(z)=\sum_{n \in \mathbb{Z}} p_{(n)}^{(i)} z^{-n-1}=\sum_{n \in \mathbb{Z}} p_{n}^{(i)} z^{-n-d_{i}-1}
$$

so that

$$
\left[D, p_{n}^{(i)}\right]=n p_{n}^{(i)}
$$

One has

$$
\left[x, p_{(n)}^{(i)}\right]=0 \quad \text { for all } x \in \widetilde{\mathfrak{g}} .
$$

5.3. The action of the Feigin-Frenkel center on objects with critical level. For $k \in \mathbb{C}$ we denote by $\mathcal{O}_{k}$ the category $\mathcal{O}$ at level $k$, i.e. the direct summand of the category $\mathcal{O}$ on which $K$ acts as multiplication with $k$. In particular, we denote by $\mathcal{O}_{\text {crit }}$ the category $\mathcal{O}$ at critical level. Let $M$ be an object of $\mathcal{O}_{\text {crit }}$. Then $M$ is naturally a graded module over the vertex algebra $V^{\text {crit }}(\mathfrak{g})$, and hence it is a graded module over its center $\mathfrak{z}(\widehat{\mathfrak{g}})$. Thus $M$ can be viewed as a graded module over the polynomial ring

$$
\mathcal{Z}=\mathbb{C}\left[p_{s}^{(i)} ; i=1,2, \ldots, \ell, s \in \mathbb{Z}\right]=\bigoplus_{n \in \mathbb{Z}} \mathcal{Z}_{n}
$$

in an obvious manner. Here $\mathcal{Z}_{n}$ is the subspace of $\mathcal{Z}$ spanned by elements $p_{n_{1}}^{\left(i_{1}\right)} \cdots p_{n_{r}}^{\left(i_{r}\right)}$ with $n_{1}+\cdots+n_{r}=n$. Set

$$
\mathcal{Z}^{-}=\mathbb{C}\left[p_{n}^{(i)} ; i=1, \ldots, \ell, n<0\right]=\bigoplus_{n \leq 0} \mathcal{Z}_{n}^{-} \subset \mathcal{Z},
$$

where $\mathcal{Z}_{n}^{-}=\mathcal{Z}^{-} \cap \mathcal{Z}_{n}$.

Theorem 5.2 ([FG06]; see also [Fre07, Theorem 9.5.3]). For any $\lambda \in \widehat{\mathfrak{h}}_{\text {crit }}^{\star}, \Delta(\lambda)$ is free over $\mathcal{Z}^{-}$. Moreover, the natural map $\mathcal{Z}_{n}^{-} \rightarrow \operatorname{Hom}(\Delta(\lambda+n \delta), \Delta(\lambda))$ is a bijection for all $n \leq 0$.

We now construct a natural map

$$
\mathcal{Z}_{n} \rightarrow \mathcal{A}_{n}
$$

Recall that $L(\delta)$ is one-dimensional. So we can choose a generator $l$ of $L(\delta)$. This gives us, for any $\widehat{\mathfrak{g}}$-module $M$, a map $M \otimes_{\mathbb{C}} L(\delta) \rightarrow M, m \otimes l \mapsto m$. Since $L(\delta)$ is trivial as a $\widetilde{\mathfrak{g}}$-module, this map is a $\widetilde{\mathfrak{g}}$-module homomorphism. By iteration we get $\widetilde{\mathfrak{g}}$-module homomorphisms $T^{n} M=M \otimes_{\mathbb{C}} L(\delta)^{\otimes n} \rightarrow M$ for all $n \geq 0$. Using the element $l^{\prime} \in L(-\delta)$ that is dual to $l$ with respect to an isomorphism $L(\delta) \otimes_{\mathbb{C}} L(-\delta) \rightarrow L(0) \cong \mathbb{C}$, we analogously get $\widetilde{\mathfrak{g}}$-module homomorphisms $T^{-n} M=$ $M \otimes_{\mathbb{C}} L(-\delta)^{\otimes n} \rightarrow M$.

Now suppose that $M$ is contained in a critical block of $\mathcal{O}$. Let $n \in \mathbb{Z}$ and $z \in \mathcal{Z}_{n}$. Then the composition of the map $T^{n} M \rightarrow M$ constructed above and the action map $z: M \rightarrow M$ now yields a $\widehat{\mathfrak{g}}$-module homomorphism $T^{n} M \rightarrow M$, as it now also commutes with the action of $D$. This gives us a natural transformation $T^{n} \rightarrow$ id and we get an element in $\mathcal{A}_{n}$ that we associate with $z$. Hence we constructed a $\operatorname{map} \mathcal{Z}_{n} \rightarrow \mathcal{A}_{n}$. 
Proof of Theorem 4.7. The action of $\mathcal{Z}_{n}$ on $\Delta(\lambda)$ factors over the action of $\mathcal{A}_{n}$. Hence Theorem 5.2 implies parts (1) and (2) of Theorem 4.7. It also implies that $\Delta(\lambda)^{-}$is spanned by $p_{-n}^{(i)} m$ with $i=1, \ldots, \ell, n>0$ and $m \in \Delta(\lambda)$. From the freeness assertion in Theorem 5.2 we deduce part (3) of Theorem 4.7 Finally, its part (4) is another Theorem of Feigin and Frenkel [FF90] which, for instance, follows from [Fre07, Proposition 9.5.1] and Theorem 5.2.

\section{Projective objects}

For a general equivalence class $\Lambda \in \widehat{\mathfrak{h}}^{\star} / \sim$ the block $\mathcal{O}_{\Lambda}$ does not contain enough projective objects (this includes, for example, all critical equivalence classes). However, there is a way to overcome this problem by restricting the set of possible weights for the modules under consideration. This means that we have to consider the truncated subcategories of $\mathcal{O}$.

6.1. The truncated categories. Let us fix a (not necessarily critical) equivalence class $\Lambda \in \widehat{\mathfrak{h}}^{\star} / \sim$. We use the following notation: We write $\{\leq \nu\}$ for the set $\left\{\nu^{\prime} \in \Lambda \mid \nu^{\prime} \leq \nu\right\}$ and use the similar notation $\{<\nu\},\{\geq \nu\}$, etc. for the analogously defined sets. We consider the topology on $\Lambda$ that is generated by the basic open sets $\{\leq \nu\}$ for $\nu \in \Lambda$. Hence a subset $\mathcal{J}$ of $\Lambda$ is open in this topology if and only if for all $\nu, \nu^{\prime} \in \Lambda$ with $\nu^{\prime} \leq \nu, \nu \in \mathcal{J}$ implies $\nu^{\prime} \in \mathcal{J}$.

Definition 6.1. For an open subset $\mathcal{J} \subset \Lambda$ we denote by $\mathcal{O}_{\Lambda}^{\mathcal{J}} \subset \mathcal{O}_{\Lambda}$ the full subcategory of objects $M$ with the property that each of its subquotients is isomorphic to $L(\lambda)$ for some $\lambda \in \mathcal{J}$.

Note that $\mathcal{O}_{\Lambda}^{\mathcal{J}}$ is an abelian category and that $\Delta(\lambda)$ and $L(\lambda)$ are contained in $\mathcal{O}_{\Lambda}^{\mathcal{J}}$ if and only if $\lambda \in \mathcal{J}$. We write $\mathcal{O}_{\Lambda}^{\leqslant \nu}$ instead of $\mathcal{O}_{\Lambda}^{\{\leqslant \nu\}}$.

6.2. Submodules, quotients and subquotients. Let $\Lambda \subset \widehat{\mathfrak{h}}^{\star}$ be an equivalence class and $\mathcal{J}$ an open subset of $\Lambda$. In this section we construct a left adjoint functor $M \mapsto M^{\mathcal{J}}$ to the inclusion functor $\mathcal{O}_{\Lambda}^{\mathcal{J}} \rightarrow \mathcal{O}_{\Lambda}$. Let us denote by $\mathcal{I}=\Lambda \backslash \mathcal{J}$ the closed complement of $\mathcal{J}$.

Definition 6.2. Let $M$ be an object in $\mathcal{O}_{\Lambda}$.

(1) We define $M_{\mathcal{I}} \subset M$ as the submodule generated by the weight spaces $M_{\nu}$ with $\nu \in \mathcal{I}$.

(2) We define $M^{\mathcal{J}}$ as the quotient of $M$ by the submodule $M_{\mathcal{I}}$.

Obviously, the map $M \mapsto M^{\mathcal{J}}$ defines a functor from $\mathcal{O}_{\Lambda} \rightarrow \mathcal{O}_{\Lambda}^{\mathcal{J}}$ that is left adjoint to the inclusion. We write $M^{\leq \nu}$ instead of $M^{\{\leq \nu\}}$ and $M^{<\nu}$ instead of $M^{\{<\nu\}}$, etc. If $\mathcal{I}^{\prime} \subset \mathcal{I} \subset \Lambda$ are closed subsets, then there is a natural inclusion $M_{\mathcal{I}^{\prime}} \subset M_{\mathcal{I}}$. If $\mathcal{J}^{\prime} \subset \mathcal{J} \subset \Lambda$ are open subsets, then there is a canonical surjective $\operatorname{map} M^{\mathcal{J}} \rightarrow M^{\mathcal{J}^{\prime}}$.

In addition to the submodules and quotient modules defined above we will also need the following subquotient modules that are associated with locally closed subsets. For any subset $\mathcal{K}$ of $\Lambda$ define

$$
\mathcal{K}_{-}:=\bigcup_{\lambda \in \mathcal{K}}\{\leq \lambda\} \text { and } \mathcal{K}_{+}:=\bigcup_{\lambda \in \mathcal{K}}\{\geq \lambda\} .
$$

Note that $\mathcal{K}_{+}$is closed and $\mathcal{K}_{-}$is open, and $\mathcal{K}$ is locally closed if $\mathcal{K}=\mathcal{K}_{-} \cap \mathcal{K}_{+}$. 
Definition 6.3. Suppose that $\mathcal{K}$ is locally closed. We define $M_{[\mathcal{K}]}$ as the image of the canonical decomposition $M_{\mathcal{K}_{+}} \hookrightarrow M \rightarrow M^{\mathcal{K}_{-}}$.

If $\mathcal{K}=\{\lambda, \ldots, \mu\}$, we write $M_{[\lambda, \ldots, \mu]}$ instead of $M_{[\{\lambda, \ldots, \mu\}]}$.

\subsection{Modules admitting a Verma flag.}

Definition 6.4. We say that $M \in \mathcal{O}_{\Lambda}$ admits a Verma flag if there is a finite filtration

$$
0=M_{0} \subset M_{1} \subset \cdots \subset M_{n}=M
$$

such that for all $i=1, \ldots, n$ the quotient $M_{i} / M_{i-1}$ is isomorphic to a Verma module $\Delta\left(\mu_{i}\right)$ for some $\mu_{i} \in \widehat{\mathfrak{h}}^{\star}$.

For each $\mu \in \widehat{\mathfrak{h}}^{\star}$ the multiplicity

$$
[M: \Delta(\mu)]:=\#\left\{i \mid \mu=\mu_{i}\right\}
$$

is independent of the chosen filtration. It is a well-known fact that $\operatorname{Ext}^{1}(\Delta(\mu), \Delta(\lambda))$ $\neq 0$ implies that $\lambda>\mu$. Hence we can find a filtration for $M$ as in the definition such that $\mu_{i}>\mu_{j}$ implies $i<j$. From this one easily gets the following lemma.

Lemma 6.5. Suppose that $M$ admits a Verma flag and suppose that $\mathcal{J} \subset \widehat{\mathfrak{h}}^{\star}$ is open, $\mathcal{I} \subset \widehat{\mathfrak{h}}^{\star}$ is closed and $\mathcal{K} \subset \widehat{\mathfrak{h}}^{\star}$ is locally closed. Then $M^{\mathcal{J}}, M_{\mathcal{I}}$ and $M_{[\mathcal{K}]}$ admit a Verma flag and for the multiplicities we have

$$
\begin{aligned}
& {\left[M^{\mathcal{J}}: \Delta(\mu)\right] }= \begin{cases}{[M: \Delta(\mu)]} & \text { if } \mu \in \mathcal{J} \\
0 & \text { else, }\end{cases} \\
& {\left[M_{\mathcal{I}}: \Delta(\mu)\right] }= \begin{cases}{[M: \Delta(\mu)]} & \text { if } \mu \in \mathcal{I} \\
0 & \text { else, }\end{cases} \\
& {\left[M_{[\mathcal{K}]}: \Delta(\mu)\right]= \begin{cases}{[M: \Delta(\mu)]} & \text { if } \mu \in \mathcal{K} \\
0 & \text { else }\end{cases} }
\end{aligned}
$$

for all $\mu \in \widehat{\mathfrak{h}}^{\star}$.

6.4. Projective objects. We say that $\mathcal{J} \subset \Lambda$ is bounded if for any $\lambda \in \mathcal{J}$ the set of $\mu \in \mathcal{J}$ with $\mu \geq \lambda$ is finite.

Theorem 6.6 ([Fie03]; cf. also [AF09]). Suppose that $\mathcal{J} \subset \Lambda$ is open and bounded and let $\lambda \in \mathcal{J}$.

(1) There exists an (up to isomorphism unique) projective cover $P^{\mathcal{J}}(\lambda)$ of $L(\lambda)$ in $\mathcal{O}_{\Lambda}^{\mathcal{J}}$.

(2) If $\mathcal{J}^{\prime} \subset \mathcal{J}$ is an open subset and $\lambda \in \mathcal{J}^{\prime}$, then we have an isomorphism $\left(P^{\mathcal{J}}(\lambda)\right)^{\mathcal{J}^{\prime}} \cong P^{\mathcal{J}^{\prime}}(\lambda)$.

(3) The object $P^{\mathcal{J}}(\lambda)$ admits a Verma flag and for each $\gamma \in \Lambda$ we have the $B G G$-reciprocity formula

$$
\left(P^{\mathcal{J}}(\lambda): \Delta(\gamma)\right)= \begin{cases}0 & \text { if } \gamma \notin \mathcal{J} \\ {[\nabla(\gamma): L(\lambda)]} & \text { if } \gamma \in \mathcal{J}\end{cases}
$$


By Lemma 6.5 and the theorem above, if $\nu, \lambda \in \Lambda$ are such that $\nu \geq \lambda$, then the module $P^{\mathcal{J}}(\lambda)_{[\nu]}$ does not depend on the open set $\mathcal{J}$ as long as $\nu \in \mathcal{J}$. We denote this object by $P(\lambda)_{[\nu]}$.

Let $\mathcal{J} \subset \Lambda$ be open and bounded and $\lambda \in \mathcal{J}$. Suppose that $\nu \in \mathcal{J}$ is a maximal element. Then we can consider $P(\lambda)_{[\nu]}$ as a subspace in $P^{\mathcal{J}}(\lambda)$. We will need the following result later.

Lemma 6.7. Under the above assumptions, the restriction map

$$
\operatorname{Hom}\left(P^{\mathcal{J}}(\lambda), \nabla(\nu)\right) \rightarrow \operatorname{Hom}\left(P(\lambda)_{[\nu]}, \nabla(\nu)\right)
$$

is a bijection.

Proof. As a first step we show that the map is injective. Suppose that $f \in$ $\operatorname{Hom}\left(P^{\mathcal{J}}(\lambda), \nabla(\nu)\right)$ is contained in the kernel. Then $P(\lambda)_{[\nu]}$ is contained in the kernel of $f$, hence $f$ factors over the map $P^{\mathcal{J}}(\lambda) \rightarrow\left(P^{\mathcal{J}}(\lambda)\right)^{\mathcal{J} \backslash\{\nu\}} \cong P^{\mathcal{J} \backslash\{\nu\}}(\lambda)$. But then it cannot contain the simple socle $L(\nu) \subset \nabla(\nu)$ in its image, so $f=0$.

Now we have

$$
\begin{aligned}
\operatorname{dim} \operatorname{Hom}\left(P^{\mathcal{J}}(\lambda), \nabla(\nu)\right) & =[\nabla(\nu): L(\lambda)] \\
& =\left(P^{\mathcal{J}}(\lambda): \Delta(\nu)\right) \\
& =\left(P(\lambda)_{[\nu]}: \Delta(\nu)\right) \\
& =\operatorname{dim} \operatorname{Hom}\left(P(\lambda)_{[\nu]}, \nabla(\nu)\right) .
\end{aligned}
$$

The last equation holds since $P(\lambda)_{[\nu]}$ is isomorphic to a direct sum of copies of $\Delta(\nu)$ and $\operatorname{dim} \operatorname{Hom}(\Delta(\nu), \nabla(\nu))=1$. Since the map referred to in the lemma is injective and since the dimensions of its source and its image coincide, it is bijective.

6.5. The Casimir operator. We call a $\widehat{\mathfrak{g}}$-module $M$ smooth if for all $m \in M$ we have $\widehat{\mathfrak{g}}_{\alpha} \cdot m=0$ for all but a finite number of positive affine roots $\alpha \in \widehat{R}$. In particular, each locally $\widehat{\mathfrak{b}}$-finite $\widehat{\mathfrak{g}}$-module is smooth. Recall that on the full subcategory $\widehat{\mathfrak{g}}-\bmod ^{s m}$ of smooth representations there is an endomorphism of the identity functor, the Casimir operator $C:$ id $\rightarrow$ id (its construction can be found, for example, in [Kac90, Section 2.5]). We will only need the following property of $C$.

Proposition 6.8. Let $\lambda \in \widehat{\mathfrak{h}}^{\star}$. Then $C$ acts on $\Delta(\lambda)$ as multiplication with the scalar $c_{\lambda}:=(\lambda+\rho, \lambda+\rho)-(\rho, \rho) \in \mathbb{C}$.

The construction of $C$ depends on $(\cdot, \cdot)$, hence there is no ambiguity in the statement of the proposition.

Let $\Lambda \subset \widehat{\mathfrak{h}}^{\star}$ be a (not necessarily critical) equivalence class. For $\lambda, \mu \in \Lambda$ we have $c_{\lambda}=c_{\mu}$, so $\Lambda$ defines a unique scalar $c_{\Lambda} \in \mathbb{C}$ such that $C$ acts by multiplication with $c_{\Lambda}$ on each Verma module in $\mathcal{O}_{\Lambda}$.

Now suppose that $\lambda, \mu \in \Lambda$ are such that they form an atom in the partially ordered set $\Lambda$, i.e. suppose that $\lambda<\mu$ and that there is no $\nu \in \Lambda$ with $\lambda<\nu<\mu$. Moreover, assume that $\bar{\lambda} \neq \bar{\mu}$. Then the object $P^{\leqslant \mu}(\lambda)$ is an extension of the Verma modules $\Delta(\lambda)$ and $\Delta(\mu)$, each occurring once (this is, by the BGG-reciprocity, equivalent to $[\Delta(\lambda): L(\lambda)]=[\Delta(\mu): L(\lambda)]=1$, which can be deduced easily from the analog of Jantzen's sum formula in the Kac-Moody case; cf. [KK79]). Hence we have a short exact sequence

$$
0 \rightarrow \Delta(\mu) \rightarrow P^{\leqslant \mu}(\lambda) \rightarrow \Delta(\lambda) \rightarrow 0 .
$$


Lemma 6.9. The endomorphism $C-c_{\Lambda} \mathrm{id}: P^{\leqslant \mu}(\lambda) \rightarrow P^{\leqslant \mu}(\lambda)$ is non-zero.

Proof. For the proof we use deformation theory; cf. Fie03 and AF09. Denote by $A=\mathbb{C}[[t]]$ the completed polynomial ring in one variable and by $Q=$ Quot $A$ its quotient field. Let us fix $\gamma \in \widehat{\mathfrak{h}}^{\star}$ with the property that $(\gamma+\rho, \lambda) \neq(\gamma+\rho, \mu)$. Let $S=S(\widehat{\mathfrak{h}})$ be the symmetric algebra over $\widehat{\mathfrak{h}}$ and consider the algebra map $\tau: S \rightarrow A$ that is determined by $\tau(h)=\gamma(h) t$ for all $h \in \widehat{\mathfrak{h}}$. This makes $A$, and hence $Q$, into a local $S$-algebra.

In Fie03 we constructed the deformed categories $\mathcal{O}_{A}$ and $\mathcal{O}_{Q}$ as full subcategories of $\widehat{\mathfrak{g}} \otimes_{\mathbb{C}} A$-mod and $\widehat{\mathfrak{g}} \otimes_{\mathbb{C}} Q$-mod. We showed that the functors $\otimes_{A} \mathbb{C}$ and $\otimes_{A} Q$ induce functors $\mathcal{O}_{A} \rightarrow \mathcal{O}$ and $\mathcal{O}_{A} \rightarrow \mathcal{O}_{Q}$. The categories $\mathcal{O}_{A}$ and $\mathcal{O}_{Q}$ contain deformed Verma modules $\Delta_{A}(\nu)$ and $\Delta_{Q}(\nu)$, respectively. On their highest weight spaces the Cartan algebra $\widehat{\mathfrak{h}}$ acts by the character $\nu+\tau$, which is considered as a linear map from $\widehat{\mathfrak{h}}$ to $A$ and $Q$, respectively. In particular, the Casimir operator $C$ acts on $\Delta_{Q}(\nu)$ as multiplication with $c_{\nu+\gamma t}=(\nu+\gamma t+\rho, \nu+\gamma t+\rho)-(\rho, \rho)$.

The analogous definition as in the non-deformed case gives truncated categories $\mathcal{O}_{A}^{\leqslant \mu}$ and $\mathcal{O}_{Q}^{\leqslant \nu}$ for all $\nu \in \widehat{\mathfrak{h}}^{\star}$. For any $\nu, \nu^{\prime} \in \widehat{\mathfrak{h}}^{\star}$ there is a projective object $P_{A}^{\leqslant \nu}\left(\nu^{\prime}\right)$ in $\mathcal{O}_{A}^{\leqslant \nu}$ such that $P_{A}^{\leqslant \nu}\left(\nu^{\prime}\right) \otimes_{A} \mathbb{C} \cong P^{\leqslant \nu}\left(\nu^{\prime}\right)$. Moreover, we have

$$
\operatorname{Hom}\left(P_{A}^{\leqslant \nu}\left(\nu_{1}^{\prime}\right), P_{A}^{\leqslant \nu}\left(\nu_{2}^{\prime}\right)\right) \otimes_{A} \mathbb{C}=\operatorname{Hom}\left(P^{\leqslant \nu}\left(\nu_{1}^{\prime}\right), P^{\leqslant \nu}\left(\nu_{2}^{\prime}\right)\right)
$$

The category $\mathcal{O}_{Q}$ is semi-simple, i.e. each object is isomorphic to a direct sum of Verma modules $\Delta_{Q}(\lambda)$. Now each $P_{A}^{\leqslant \nu}\left(\nu^{\prime}\right) \otimes_{A} Q$ admits a $Q$-deformed Verma flag, hence it splits into a direct sum of $Q$-deformed Verma modules. The Verma multiplicities of $P_{A}^{\leqslant \nu}\left(\nu^{\prime}\right)$, of $P^{\leqslant \nu}\left(\nu^{\prime}\right)$ and of $P_{A}^{\leqslant \nu}\left(\nu^{\prime}\right) \otimes_{A} Q$ coincide.

Now let $\lambda, \mu \in \Lambda$ be as in the statement of the lemma. Note that $c_{\lambda+\gamma t} \equiv c_{\mu+\gamma t}$ $\bmod t$, but our choice of $\gamma$ implies $c_{\lambda+\gamma t} \neq c_{\mu+\gamma t}$. Let us suppose that the action of $C-c_{\Lambda}$ id on $P^{\leqslant \mu}(\lambda)$ was zero. From the above, our assumptions on $\lambda$ and $\mu$ and the Jantzen sum formula we get an inclusion

$$
P_{A}^{\leqslant \mu}(\lambda) \subset P_{A}^{\leqslant \mu}(\lambda) \otimes_{A} Q \cong \Delta_{Q}(\lambda) \oplus \Delta_{Q}(\mu)
$$

On each of the modules above the Casimir operator acts. Our assumptions imply that the image of the action of $C-c_{\Lambda}$ id on the module on the left is contained in $t P_{A}^{\leqslant \mu}(\lambda)$, hence $t^{-1}\left(C-c_{\Lambda}\right.$ id $)$ is a well-defined operator on $P_{A}^{\leqslant \mu}(\lambda)$. On the module on the right this operator acts diagonally with eigenvalues in $\mathbb{C}[[t]]$ which are distinct modulo $t$. Hence $P_{A}^{\leqslant \mu}(\lambda)$ decomposes according to the inclusion above, which clearly cannot be the case.

Lemma 6.10. Suppose that $\lambda, \mu \in \Lambda$ are as above and that

$$
0 \rightarrow \Delta(\mu) \rightarrow M \rightarrow X \rightarrow 0
$$

is a short exact sequence, where $X$ is a module of highest weight $\lambda$. If $C$ acts on $M$ as a scalar, then there is a submodule $Y$ of $M$ with highest weight $\lambda$ that maps surjectively onto $X$. 
Proof. Since $X$ is a module of highest weight $\lambda$ and since the weights of $M$ are smaller then or equal to $\mu$, there is a map $f: P^{\leqslant \mu}(\lambda) \rightarrow M$ such that the composition $P^{\leqslant \mu}(\lambda) \rightarrow M \rightarrow X$ is surjective. There is a commutative diagram

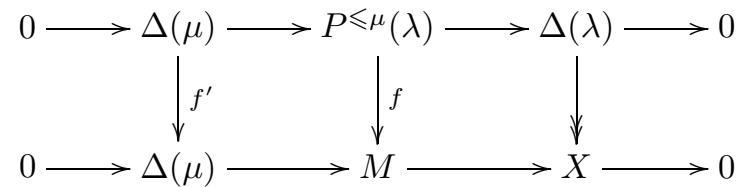

with exact rows. In order to prove the lemma it is enough to show that $\Delta(\mu) \subset$ $P^{\leqslant \mu}(\lambda)$ is in the kernel of $f$, i.e. that the left vertical map $f^{\prime}$ is zero. Since any endomorphism of a Verma module is either zero or injective, it suffices to show that $f^{\prime}$ is not injective.

By assumption, the Casimir element $C$ acts on $M$ by a scalar, which has to be $c_{\Lambda}$. So $C-c_{\Lambda}$ id acts on $M$ by zero, so $\left(C-c_{\Lambda}\right.$ id $) P^{\leqslant \mu}(\lambda)$ is in the kernel of $f$. Since $C-c_{\Lambda}$ id acts by zero on $\Delta(\lambda)$, we have $\left(C-c_{\Lambda}\right.$ id $) P^{\leqslant \mu}(\lambda) \subset \Delta(\mu) \subset P \leqslant \mu(\lambda)$. By the previous lemma, $\left(C-c_{\Lambda}\right.$ id $) P^{\leqslant \mu}(\lambda)$ is non-zero, so the kernel of $f^{\prime}$ is not trivial. Hence $f^{\prime}$ is not injective, hence it must be zero, which is what we wanted to show.

6.6. The action of $\mathcal{A}$ on projective objects. Let us now fix a critical equivalence class $\Lambda \subset \widehat{\mathfrak{h}}^{\star}$. Let $\lambda \in \Lambda$ and $n \geq 0$. In this section we study the action of $\mathcal{A}_{n}$ on $P^{\leqslant \lambda}(\lambda-n \delta)$, i.e. we want to study the map

$$
\mathcal{A}_{n} \rightarrow \operatorname{Hom}\left(T^{n} P^{\leqslant \lambda}(\lambda-n \delta), P^{\leqslant \lambda}(\lambda-n \delta)\right) .
$$

For the ease of notation let us fix an identification $T^{n} P^{\leqslant \lambda}(\lambda-n \delta) \cong P^{\leqslant \lambda+n \delta}(\lambda)$. Each map $f: P^{\leqslant \lambda+n \delta}(\lambda) \rightarrow P^{\leqslant \lambda}(\lambda-n \delta)$ factors over the map $P^{\leqslant \lambda+n \delta}(\lambda) \rightarrow$ $P \leqslant \lambda(\lambda)$. The latter module is isomorphic to $\Delta(\lambda)$, hence the image of the map $f$ must be contained in $P(\lambda-n \delta)_{[\lambda]} \subset P^{\leqslant \lambda}(\lambda-n \delta)$. So the map $f$ induces a unique map $f^{\prime}: P \leqslant \lambda(\lambda) \rightarrow P(\lambda-n \delta)_{[\lambda]}$ such that the following diagram commutes:

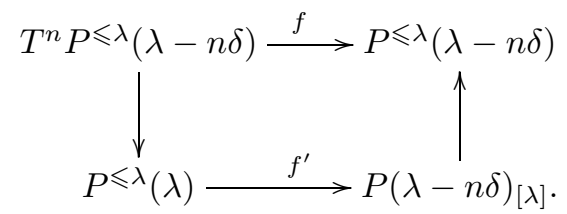

The next proposition is one of the principal technical ingredients in the proof of our main theorem.

Proposition 6.11. Suppose that $n \geq 0$ is such that $[\Delta(\lambda): L(\lambda-n \delta)]=p(n)$. Then the action map

$$
\begin{aligned}
\mathcal{A}_{n} & \rightarrow \operatorname{Hom}\left(T^{n} P^{\leqslant \lambda}(\lambda-n \delta), P^{\leqslant \lambda}(\lambda-n \delta)\right) \\
& \cong \operatorname{Hom}\left(P^{\leqslant \lambda}(\lambda), P(\lambda-n \delta)_{[\lambda]}\right)
\end{aligned}
$$

is surjective.

Proof. Note that $P(\lambda) \leqslant \lambda \cong \Delta(\lambda)$ and that $P(\lambda-n \delta)_{[\lambda]}$ is isomorphic to a direct sum of $\left(P(\lambda-n \delta)_{[\lambda]}: \Delta(\lambda)\right)$-copies of $\Delta(\lambda)$. By our assumption and the BGGreciprocity, this number is $p(n)$. Hence the spaces on the right hand side of our map are of dimension $p(n)$. 
Now the following Lemma 6.13 shows that the image of the action map $\mathcal{A}_{n} \rightarrow$ $\operatorname{Hom}\left(T^{n} P^{\leqslant \lambda}(\lambda-n \delta), P^{\leqslant \lambda}(\lambda-n \delta)\right)$ is of dimension $p(n)$. From this we deduce our claim.

6.7. A duality on $\mathcal{A}$. Let $\Lambda \subset \widehat{\mathfrak{h}}^{\star}$ again be a critical equivalence class. In this section we define an algebra involution

$$
\mathrm{D}: \mathcal{A} \rightarrow \mathcal{A}
$$

which maps $\mathcal{A}_{n}$ into $\mathcal{A}_{-n}$.

Fix $n \in \mathbb{Z}$ and choose $z \in \mathcal{A}_{n}$. We define $\mathrm{D} z \in \mathcal{A}_{-n}$ as follows. Let $M \in \mathcal{O}_{\Lambda}^{f}:=$ $\mathcal{O}^{f} \cap \mathcal{O}_{\Lambda}$ and let $M^{\star} \in \mathcal{O}_{\Lambda}$ be its restricted dual. Then $z$ defines a homomorphism $z^{M^{\star}}: T^{n} M^{\star} \rightarrow M^{\star}$. The dual of this map is a homomorphism $\left(z^{M^{\star}}\right)^{\star}: M \rightarrow$ $T^{n} M$.

Definition 6.12. For $z \in \mathcal{A}_{n}$ and $M \in \mathcal{O}_{\Lambda}^{f}$ define the map

$$
(\mathrm{D} z)^{M}:=T^{-n}\left(z^{M^{\star}}\right)^{\star}: T^{-n} M \rightarrow M
$$

One immediately checks that we get a natural transformation $\mathrm{D} z:\left.T^{-n}\right|_{\mathcal{O}_{\Lambda}^{f}} \rightarrow$ $\operatorname{id}_{\mathcal{O}_{\Lambda}^{f}}$. As $\mathcal{O}_{\Lambda}$ is filtered by the truncated categories, and as each indecomposable projective object in a truncated category is also contained in $\mathcal{O}_{\Lambda}^{f}$, this induces a natural transformation $\mathrm{D} z: T^{n} \rightarrow$ id between the functors on the whole block $\mathcal{O}_{\Lambda}$, hence an element in $\mathcal{A}_{-n}$.

Now we prove the statement that remained open in the proof of Proposition 6.11. Fix $\lambda \in \Lambda$ and $n \geq 0$.

Lemma 6.13. For $z \in \mathcal{A}_{n}$ the following holds:

$$
(\mathrm{D} z)^{\Delta(\lambda)} \neq 0 \text { if and only if } z^{P^{\leqslant \lambda}(\lambda-n \delta)} \neq 0 .
$$

In particular, the image of the map $\mathcal{A}_{n} \rightarrow \operatorname{Hom}\left(T^{n} P^{\leqslant \lambda}(\lambda-n \delta), P^{\leqslant \lambda}(\lambda-n \delta)\right)$ is of dimension $p(n)$.

Proof. By the definition of the duality we have

$$
(\mathrm{D} z)^{\Delta(\lambda)} \neq 0 \text { if and only if } z^{\nabla(\lambda)} \neq 0 .
$$

For each homomorphism $g: P^{\leqslant \lambda}(\lambda-n \delta) \rightarrow \nabla(\lambda)$ there is a commutative diagram

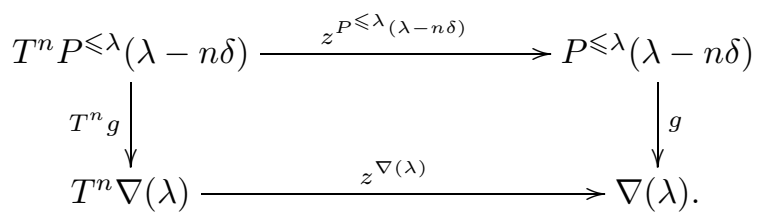

The strategy of the proof is the following. Suppose that $z^{P^{\leqslant \lambda}(\lambda-n \delta)} \neq 0$. We show that there is a map $g$ such that the top right composition in the diagram above is non-zero. From this we deduce that $z^{\nabla(\lambda)} \neq 0$. We show that $z^{\nabla(\lambda)} \neq 0$ implies $z^{P^{\leqslant \lambda}(\lambda-n \delta)} \neq 0$ in a similar way. 
So suppose that $z^{P^{\leqslant \lambda}(\lambda-n \delta)} \neq 0$. We have already seen that there is a unique map $b: P^{\leqslant \lambda}(\lambda) \rightarrow P(\lambda-n \delta)_{[\lambda]}$ such that the following diagram commutes:

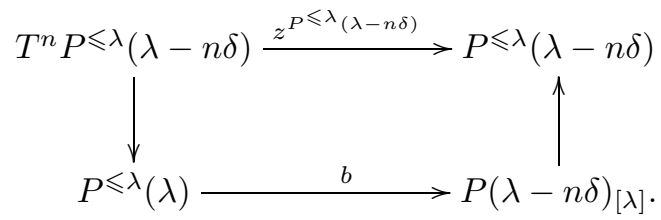

Now $P^{\leqslant \lambda}(\lambda)$ is isomorphic to $\Delta(\lambda)$ and $P(\lambda-n \delta)_{[\lambda]}$ is a direct sum of various copies of $\Delta(\lambda)$. Hence we can find a map $g^{\prime}: P(\lambda-n \delta)_{[\lambda]} \rightarrow \nabla(\lambda)$ such that the composition $P \leqslant \lambda(\lambda) \stackrel{b}{\rightarrow} P(\lambda-n \delta)_{[\lambda]} \stackrel{g^{\prime}}{\rightarrow} \nabla(\lambda)$ is non-zero. By Lemma 6.7 the map $g^{\prime}$ admits a lift $g: P^{\leqslant \lambda}(\lambda-n \delta) \rightarrow \nabla(\lambda)$. Diagrammatically, the situation now looks as follows:

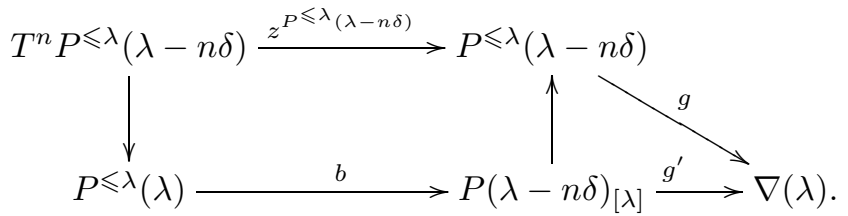

If we plug in the map $g$ that we just obtained in the first diagram above, then the top right composition is non-zero, hence so is the bottom left composition. In particular, $z^{\nabla(\lambda)} \neq 0$. This is the first part of the proof.

Now suppose that $z^{\nabla(\lambda)} \neq 0$. Then the image of $z^{\nabla(\lambda)}$ contains the unique simple submodule $L(\lambda)$ of $\nabla(\lambda)$. Since $T^{n} P^{\leqslant \lambda}(\lambda-n \delta) \cong P^{\leqslant \lambda+n \delta}(\lambda)$ is a projective cover of $L(\lambda)$ in $\mathcal{O}^{\leqslant \lambda+n \delta}$, and since $T^{n} \nabla(\lambda) \cong \nabla(\lambda+n \delta)$ is contained in the latter category, we can find a map $g^{\prime}: T^{n} P^{\leqslant \lambda}(\lambda-n \delta) \rightarrow T^{n} \nabla(\lambda)$ such that $z^{\nabla(\lambda)} \circ g^{\prime}$ is non-zero. For $g:=T^{-n} g^{\prime}$, the bottom left composition in the first diagram in this proof is non-zero, hence so is the top right composition. In particular, $z^{P^{\leqslant \lambda}(\lambda-n \delta)} \neq 0$.

The last statement of the lemma follows from the previous result and Theorem 4.7.

6.8. A variant for the subgeneric cases. We will also need the following variant of Proposition 6.11 in the case that $\Lambda$ is critical and subgeneric. Suppose that $\alpha$ is the positive finite root with $\bar{\Lambda}=\left\{\bar{\lambda}, s_{\alpha} \cdot \bar{\lambda}\right\}$. Fix $\lambda \in \Lambda$ and $n \geq 0$. We study the action of $\mathcal{A}_{n}$ on the projective cover $P^{\leqslant \alpha \uparrow \lambda}(\lambda-n \delta)$, i.e. we now consider the map

$$
\mathcal{A}_{n} \rightarrow \operatorname{Hom}\left(T^{n} P^{\leqslant \alpha \uparrow \lambda}(\lambda-n \delta), P^{\leqslant \alpha \uparrow \lambda}(\lambda-n \delta)\right) .
$$

Again, let us fix an isomorphism $T^{n} P^{\leqslant \alpha \uparrow \lambda}(\lambda-n \delta) \cong P^{\leqslant \alpha \uparrow \lambda+n \delta}(\lambda)$. As before we see that each map $f: P^{\leqslant \alpha \uparrow \lambda+n \delta}(\lambda) \rightarrow P^{\leqslant \alpha \uparrow \lambda}(\lambda-n \delta)$ induces a map $f^{\prime}: P^{\leqslant \alpha \uparrow \lambda}(\lambda) \rightarrow$ $P(\lambda-n \delta)_{[\lambda, \alpha \uparrow \lambda]}$ such that the following diagram commutes:

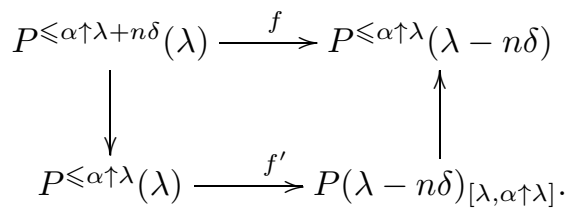

Note that $P \leqslant \alpha \uparrow \lambda(\lambda)$ is a non-split extension of the Verma modules $\Delta(\lambda)$ and $\Delta(\alpha \uparrow \lambda)$, as $\{\lambda, \alpha \uparrow \lambda\} \subset \Lambda$ is an atom. The module $P(\lambda-n \delta)_{[\lambda, \alpha \uparrow \lambda]}$ is an extension of $[\Delta(\lambda): L(\lambda-n \delta)]$ many copies of $\Delta(\lambda)$ and $[\Delta(\alpha \uparrow \lambda): L(\lambda-n \delta)]$ many copies of $\Delta(\alpha \uparrow \lambda)$. 
Let us assume that $[\Delta(\alpha \uparrow \lambda): L(\lambda-n \delta)]=[\Delta(\lambda): L(\lambda-n \delta)]=p(n)$. Then $P(\lambda-n \delta)_{[\lambda, \alpha \uparrow \lambda]}$ is a direct sum of $p(n)$ non-split extensions of the Verma modules $\Delta(\lambda)$ and $\Delta(\alpha \uparrow \lambda)$ (the extensions are non-split by projectivity).

Let us consider the composition

$$
\begin{aligned}
\mathcal{A}_{n} & \rightarrow \operatorname{Hom}\left(T^{n} P^{\leqslant \alpha \uparrow \lambda}(\lambda-n \delta), P^{\leqslant \alpha \uparrow \lambda}(\lambda-n \delta)\right) \\
& \rightarrow \operatorname{Hom}\left(P(\lambda)_{[\alpha \uparrow \lambda]}, P(\lambda-n \delta)_{[\alpha \uparrow \lambda]}\right),
\end{aligned}
$$

where the last map is induced by the functor $(\cdot)_{[\alpha \uparrow \lambda]}$.

Proposition 6.14. Suppose that $[\Delta(\alpha \uparrow \lambda): L(\lambda-n \delta)]=[\Delta(\lambda): L(\lambda-n \delta)]=$ $p(n)$. Then the composition $\mathcal{A}_{n} \rightarrow \operatorname{Hom}\left(P(\lambda)_{[\alpha \uparrow \lambda]}, P(\lambda-n \delta)_{[\alpha \uparrow \lambda]}\right)$ constructed above is surjective.

Proof. Consider the homomorphisms

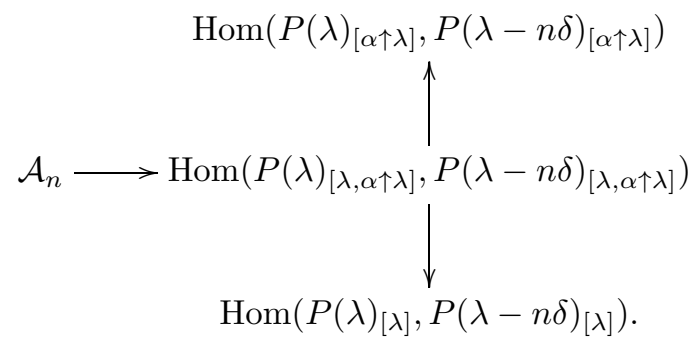

By Proposition 6.11, the lower composition is surjective. But the kernel of the upper composition is contained in the kernel of the lower composition, as $P(\lambda-n \delta)_{[\alpha \uparrow \lambda, \lambda]}$ is a direct sum of non-split extensions of $\Delta(\alpha \uparrow \lambda)$ and $\Delta(\lambda)$. Since the spaces on the top and the bottom share the same dimension, the upper composition is also surjective.

\section{The BRST COHOMOLOGY}

To prove Theorem 4.9 we need a result from [Ara07, which we explain below.

7.1. The BRST cohomology associated with the quantized DrinfeldSokolov reduction. Denote by $\mathfrak{n}_{-}:=\bigoplus_{\alpha \in R^{+}} \mathfrak{g}_{-\alpha}$ the nilpotent subalgebra of $\mathfrak{g}$ corresponding to the set of negative roots. Let $\Psi$ be a non-degenerate character of $\mathfrak{n}_{-}$in the sense of Kostant [Kos78, i.e.

$$
\Psi(x)=k\left(x, e_{\text {prin }}\right)
$$

for some principal nilpotent element $e_{\text {prin }}$ of $\mathfrak{g}$ in $\mathfrak{n}_{+}:=\bigoplus_{\alpha \in R^{+}} \mathfrak{g}_{\alpha}$. We extend $\Psi$ to the character $\widehat{\Psi}$ of $\mathfrak{n}_{-}\left[t, t^{-1}\right]:=\mathfrak{n}_{-} \otimes \mathbb{C}\left[t, t^{-1}\right] \subset \widehat{\mathfrak{g}}$ by setting

$$
\widehat{\Psi}\left(u \otimes t^{n}\right)=\Psi(u) \delta_{n, 0}
$$

for $u \in \mathfrak{n}_{-}, n \in \mathbb{Z}$. Let $\mathbb{C}_{\widehat{\Psi}}$ be the corresponding one-dimensional representation of $\mathfrak{n}_{-}\left[t, t^{-1}\right]$.

Set

$$
H^{i}(M):=H^{\frac{\infty}{2}+i}\left(\mathfrak{n}_{-}\left[t, t^{-1}\right], M \otimes \mathbb{C}_{\widehat{\Psi}}\right)
$$

for $M \in \mathcal{O}_{\text {crit }}$ and $i \in \mathbb{Z}$. Here, $M \otimes \mathbb{C}_{\widehat{\Psi}}$ is considered as an $\mathfrak{n}_{-}\left[t, t^{-1}\right]$-module by the tensor product action, and $H^{\frac{\infty}{2}+\bullet}\left(\mathfrak{n}_{-}\left[t, t^{-1}\right], M \otimes \mathbb{C}_{\widehat{\Psi}}\right)$ is the semi-infinite $\mathfrak{n}_{-}\left[t, t^{-1}\right]$-cohomology Fei84] with coefficients in $M \otimes \mathbb{C}_{\widehat{\Psi}}$. The cohomology $H^{\bullet}(M)$ is defined by the semi-infinite analogue of the Chevalley-Eilenberg complex: Let $\mathcal{C l}$ 
be the unital superalgebra generated by odd elements $\psi_{\alpha}(n)$ for $\alpha \in R, n \in \mathbb{Z}$, with the relations

$$
\psi_{\alpha}(m) \psi_{\beta}(n)+\psi_{\beta}(n) \psi_{\alpha}(m)=\delta_{m+n, 0} \delta_{\alpha+\beta, 0} .
$$

Let $\Lambda^{\frac{\infty}{2}+}$ be the irreducible representation of $\mathcal{C l}$ generated by the vector 1 such that

$$
\psi_{\alpha}(n) \mathbf{1}=0 \quad \text { if } \alpha+n \delta \in \widehat{R}^{+} .
$$

The space $\Lambda^{\frac{\infty}{2}+\bullet}$ is graded by charge: $\bigwedge^{\frac{\infty}{2}+\bullet}=\bigoplus_{i \in \mathbb{Z}} \Lambda^{\frac{\infty}{2}+i}$, where the charges of $\mathbf{1}, \psi_{\alpha}(n)$ and $\psi_{-\alpha}(n)$ for $\alpha \in R^{+}, n \in \mathbb{Z}$, are 0,1 , and -1 , respectively. Also, we view $\bigwedge^{\frac{\infty}{2}+\bullet}$ as an $\widehat{\mathfrak{h}}$-module on which $h \in \widehat{\mathfrak{h}}$ acts as $h \mathbf{1}=0$ and $\left[h, \psi_{\alpha}(n)\right]=$ $\langle\alpha+n \delta, h\rangle \psi_{\alpha}(n)$.

Let $M$ be an object of $\mathcal{O}$. Set

$$
C^{\bullet}(M):=M \otimes \bigwedge^{\frac{\infty}{2}+\bullet}=\bigoplus_{i \in \mathbb{Z}} C^{i}(M), \quad C^{i}(M)=M \otimes \bigwedge^{\frac{\infty}{2}+i} .
$$

Define an odd operator $Q$ of charge 1 on $C^{\bullet}(M)$ by

$$
\begin{aligned}
Q:= & \sum_{\substack{\alpha \in R^{-} \\
n \in \mathbb{Z}}}\left(x_{\alpha} \otimes t^{-n}+\widehat{\Psi}\left(x_{\alpha} \otimes t^{-n}\right)\right) \otimes \psi_{\alpha}(n) \\
& -\frac{1}{2} \sum_{\substack{\alpha, \beta, \gamma \in R^{-} \\
k, l \in \mathbb{Z}^{-}}} c_{\alpha, \beta}^{\gamma} \operatorname{id}_{M} \otimes \psi_{-\alpha}(-k) \psi_{-\beta}(-l) \psi_{\gamma}(k+l),
\end{aligned}
$$

where $x_{\alpha}$ is a (fixed) root vector in $\mathfrak{g}_{\alpha}$ for any $\alpha \in R^{-}$and $\left[x_{\alpha}, x_{\beta}\right]=\sum_{\gamma} c_{\alpha, \beta}^{\gamma} x_{\gamma}$. The operator $Q$ is well-defined because $M \in \mathcal{O}$. One has

$$
Q^{2}=0 \text {. }
$$

Therefore, $\left(C^{\bullet}(M), Q\right)$ is a cochain complex. The space $H^{\bullet}(M)$ is by definition the cohomology of the complex $\left(C^{\bullet}(M), Q\right)$.

Set $C^{\bullet}(M)_{d}:=\left\{c \in C^{\bullet}(M) \mid(D \otimes 1+1 \otimes D) c=d c\right\}$. One has $C^{\bullet}(M)=$ $\bigoplus_{d \in \mathbb{C}} C^{\bullet}(M)_{d}$. Because the operator $Q$ obviously preserves each subspace $C^{\bullet}(M)_{d}$, $H^{\bullet}(M)$ is also graded by the diagonal action of $D$ :

$$
H^{\bullet}(M)=\bigoplus_{d \in \mathbb{C}} H^{\bullet}(M)_{d} .
$$

7.2. The functor $F$. Let $p$ be an element of $\mathfrak{z}(\widehat{\mathfrak{g}})$. For each $n \in \mathbb{Z}$, the operator $p_{(n)} \otimes 1$ commutes with the action of $Q$ on $C^{\bullet}(M)$. Therefore, for each $i \in \mathbb{Z}$, $H^{i}(M)$ is naturally a graded module over the commutative vertex algebra $\mathfrak{z}(\widehat{\mathfrak{g}})$, and thus can be considered as a graded $\mathcal{Z}$-module. Denote by $F$ the functor

$$
\mathcal{O}_{\text {crit }} \rightarrow \mathcal{Z} \text {-Mod, } \quad M \mapsto H^{0}(M),
$$

where $\mathcal{Z}$-Mod is the category of graded $\mathcal{Z}$-modules.

Set

$$
\operatorname{ch}_{q} F(M):=\sum_{d \in \mathbb{C}} q^{-d} \operatorname{dim}_{\mathbb{C}} F(M)_{d}
$$

for a finitely generated object $M$ of $\mathcal{O}_{\text {crit }}$, where $F(M)_{d}=H^{0}(M)_{d}$. 
Theorem 7.1 (Ara07]).

(1) One has $H^{i}(M)=0$ for all $i \neq 0$ and $M \in \mathcal{O}_{\text {crit }}$. In particular, the functor $F$ is exact.

(2) Let $\lambda \in \widehat{\mathfrak{h}}_{\text {crit }}^{\star}$. One has the following:

$$
\begin{aligned}
& \operatorname{ch}_{q} F(\Delta(\lambda))=q^{-\langle\lambda, D\rangle} \prod_{j \geq 1}\left(1-q^{j}\right)^{- \text {rank } g} \\
& \operatorname{ch}_{q} F(L(\lambda))= \begin{cases}q^{-\langle\lambda, D\rangle} & \text { if } \lambda \text { is anti-dominant }, \\
0 & \text { otherwise. }\end{cases}
\end{aligned}
$$

Remark 7.2. In general, the correspondence $M \mapsto H^{0}(M)$ defines a functor from $\mathcal{O}_{k}$ to the category of graded modules over the $W$-algebra $\mathscr{W}^{k}(\mathfrak{g})$ associated with $\mathfrak{g}$ at level $k$, which coincides FF92] with $\mathfrak{z}(\widehat{\mathfrak{g}})$ if the level $k$ is critical. In Ara07, it was proved that the functor $H^{0}(?)$ is exact and $H^{0}(L(\lambda))$ is zero or irreducible for any $\lambda$ at any level $k$.

\section{THE PROOF OF THE MAIN THEOREM}

We have collected all the ingredients for the proof of our main theorem, Theorem 4.9. We start with claim (1). Let us state it again:

Theorem 8.1. Let $\Lambda \subset \widehat{\mathfrak{h}}^{\star}$ be a critical equivalence class and suppose that $\nu \in \Lambda$ is anti-dominant. Then for all $w \in \mathcal{W}(\Lambda)$ and $n \geq 0$ we have

$$
[\Delta(w \cdot \nu): L(\nu-n \delta)]=p(n) .
$$

Proof. By Theorem 7.1. (1), the functor $F$ is exact. Hence we have

$$
\begin{aligned}
\operatorname{ch}_{q} F(\Delta(w . \nu)) & =\sum_{\gamma \in \Lambda}[\Delta(w \cdot \nu): L(\gamma)] \operatorname{ch}_{q} F(L(\gamma)) \\
& =\sum_{\substack{\gamma \in \Lambda \\
\gamma \text { anti-dominant }}}[\Delta(w \cdot \nu): L(\gamma)] \operatorname{ch}_{q} F(L(\gamma)) .
\end{aligned}
$$

Note that $\gamma \in \Lambda$ is anti-dominant if and only if $\gamma=\nu+r \delta$ for some $r \in \mathbb{Z}$. Since $\langle w . \nu, D\rangle=\langle\nu, D\rangle$ for all $w \in \mathcal{W}$ (as $\langle\alpha, D\rangle=0$ for all finite roots $\alpha$ ), the claim now follows directly from Theorem 7.1 (2).

It remains to prove part (2) of Theorem 4.9, Let us recall the statement:

Theorem 8.2. Let $\Lambda$ be a subgeneric critical equivalence class and let $\lambda \in \Lambda$ be dominant. Denote by $\alpha$ the positive finite root with $\left\{\bar{\lambda}, s_{\alpha} \cdot \bar{\lambda}\right\}$. Then we have, for all $n \geq 0$,

$$
[\Delta(\lambda): L(\lambda-n \delta)]=[\Delta(\alpha \uparrow \lambda): L(\lambda-n \delta)]=p(n) .
$$

For the proof of the above statement we need the following result.

Lemma 8.3. Suppose that $\lambda, \Lambda$ and $\alpha$ are as in Theorem 8.2. For any $n \geq 0$ we then have

$$
\operatorname{dim} \Delta(\lambda)_{\lambda-n \delta}^{\widehat{\mathfrak{n}}_{+}}=\operatorname{dim} \Delta(\alpha \uparrow \lambda)_{\lambda-n \delta}^{\widehat{\mathfrak{n}}_{+}}=p(n) .
$$

Proof. Note that $\operatorname{dim} \Delta(\lambda)_{\lambda-n \delta}^{\widehat{\mathfrak{n}}_{+}}=\operatorname{dim} \operatorname{Hom}(\Delta(\lambda-n \delta), \Delta(\lambda))$, so Theorem 4.7 yields $\operatorname{dim} \Delta(\lambda)_{\lambda-n \delta}^{\widehat{\mathfrak{n}}_{+}}=p(n)$. 
Let $\gamma \in \Lambda$ be arbitrary. The Jantzen sum formula yields $[\Delta(\gamma): L(\alpha \downarrow \gamma)]=1$ (note that $\alpha \downarrow \gamma$ is maximal in the set $\{\alpha \downarrow \gamma+n \delta \mid[\Delta(\gamma): L(\alpha \downarrow \gamma+n \delta)] \neq$ $0\})$. Hence $\operatorname{dim} \operatorname{Hom}(\Delta(\alpha \downarrow \gamma), \Delta(\gamma))=1$. Since any non-trivial homomorphism between Verma modules is injective, we can view $\Delta(\alpha \downarrow \gamma)$ as a uniquely defined submodule in $\Delta(\gamma)$. In particular, we have a chain of inclusions

$$
\Delta(\lambda) \subset \Delta(\alpha \uparrow \lambda) \subset \Delta\left(\alpha \uparrow^{2} \lambda\right) \subset \Delta\left(\alpha \uparrow^{3} \lambda\right) .
$$

From now on we view each of these modules as a submodule of all the modules appearing on its right (note that $\operatorname{dim} \operatorname{Hom}\left(\Delta(\lambda), \Delta\left(\alpha \uparrow^{2} \lambda\right)\right)>1$ in general). By Theorem 5.2, there is an element $z \in \mathcal{Z}^{-}$, uniquely defined up to multiplication with a scalar in $\mathbb{C}^{\times}$, such that $\Delta(\lambda)=z \Delta\left(\alpha \uparrow^{2} \lambda\right)$. It is easy to see that this implies $\Delta(\alpha \uparrow \lambda)=z \Delta\left(\alpha \uparrow^{3} \lambda\right)$.

We fix non-zero vectors $v_{\alpha \uparrow^{2} \lambda}$ and $v_{\alpha \uparrow^{3} \lambda}$ of highest weight in $\Delta\left(\alpha \uparrow^{2} \lambda\right)$ and $\Delta\left(\alpha \uparrow^{3} \lambda\right)$, respectively. We consider both as elements in the free $\mathcal{Z}^{-}$-module $\Delta\left(\alpha \uparrow^{3} \lambda\right)$. As $\mathcal{Z}^{-}$is a graded polynomial ring (in infinitely many variables), as $\mathcal{Z}_{0}^{-} \cong \mathbb{C}$ and as $v_{\alpha \uparrow^{3} \lambda}$ and $v_{\alpha \uparrow^{2} \lambda}$ are not contained in $\left(\bigoplus_{n<0} \mathcal{Z}_{n}^{-}\right) \Delta\left(\alpha \uparrow^{3} \lambda\right)$, we can extend the set $\left\{v_{\alpha \uparrow^{2} \lambda}, v_{\alpha \uparrow^{3} \lambda}\right\}$ to a $\mathcal{Z}^{-}$-basis of $\Delta\left(\alpha \uparrow^{3} \lambda\right)$. In particular, if $v \in \Delta(\alpha \uparrow \lambda)=z \Delta\left(\alpha \uparrow^{3} \lambda\right)$ is of the form $\tilde{z} v_{\alpha \uparrow^{2} \lambda}$ for some $\tilde{z} \in \mathcal{Z}^{-}$, then $\tilde{z}$ is divisible by $z$ in $\mathcal{Z}^{-}$.

Now let $v \in \Delta(\alpha \uparrow \lambda)_{\lambda-n \delta}^{\widehat{\mathfrak{n}}_{+}}$. Then, again by Theorem [5.2, $v=\tilde{z} v_{\alpha \uparrow^{2} \lambda}$ for some $\tilde{z} \in \mathcal{Z}^{-}$. As we have observed above, $\tilde{z}$ is divisible by $z$ in $\mathcal{Z}^{-}$, hence $v$ is contained in $\Delta(\lambda)$. Hence

$$
\Delta(\alpha \uparrow \lambda)_{\lambda-n \delta}^{\widehat{\mathfrak{n}}_{+}}=\Delta(\lambda)_{\lambda-n \delta}^{\widehat{\mathfrak{n}}_{+}},
$$

and we conclude $\operatorname{dim} \Delta(\alpha \uparrow \lambda)_{\lambda-n \delta}^{\widehat{\mathfrak{n}}_{+}}=p(n)$ from what we have shown earlier.

Proof of Theorem 8.2. Note first that the claimed identities are equivalent to the identities

$$
\begin{aligned}
{[\Delta(\lambda): L(\lambda-n \delta)] } & =\operatorname{dim} \Delta(\lambda)_{\lambda-n \delta}^{\widehat{\mathfrak{n}}_{+}}, \\
{[\Delta(\alpha \uparrow \lambda): L(\lambda-n \delta)] } & =\operatorname{dim} \Delta(\alpha \uparrow \lambda)_{\lambda-n \delta}^{\widehat{\mathfrak{n}}_{+}}
\end{aligned}
$$

as the right hand sides both equal $p(n)$ by Lemma 8.3 . Hence we want to prove that each simple subquotient with highest weight $\lambda-n \delta$ of $\Delta(\lambda)$ or $\Delta(\alpha \uparrow \lambda)$ corresponds to a primitive vector. As $\Delta(\alpha \uparrow \lambda)$ can be considered as a submodule of $\Delta\left(\alpha \uparrow^{2} \lambda\right)$ and as $\alpha \uparrow^{2} \lambda$ is dominant again, it is enough to prove the above claim for the simple subquotients of $\Delta(\lambda)$, i.e. it is enough to prove that $[\Delta(\lambda): L(\lambda-n \delta)]=$ $\operatorname{dim} \Delta(\lambda)_{\lambda-n \delta}^{\widehat{\mathfrak{n}}_{+}}$.

We prove this by induction on the number $n$. The case $n=0$ is easy to settle: we certainly have $[\Delta(\lambda): L(\lambda)]=1=\operatorname{dim} \Delta(\lambda)_{\lambda}^{\widehat{\mathfrak{n}}^{+}}$. So let us assume that $n>0$ and that

$$
[\Delta(\lambda): L(\lambda-l \delta)]=\operatorname{dim} \Delta(\lambda)_{\lambda-l \delta}^{\widehat{\mathfrak{n}}^{+}}
$$

holds for all $l<n$. Let

$$
V(\lambda):=\Delta(\lambda) / \Delta(\alpha \downarrow \lambda)
$$

be the Weyl module with highest weight $\lambda$ (again we consider $\Delta(\alpha \downarrow \lambda)$ as a uniquely define submodule in $\Delta(\lambda))$. Now we prove the following statement:

(1) We have $[V(\lambda): L(\lambda-n \delta)]=\operatorname{dim} V(\lambda)_{\lambda-n \delta}^{\widehat{\mathfrak{n}}_{+}}$. 
Suppose that $X$ is a submodule of $V(\lambda)$ such that there exists a surjection $f: X \rightarrow$ $L(\lambda-n \delta)$. In order to prove claim (1) it is enough to show that there is a map $g: \Delta(\lambda-n \delta) \rightarrow X$ such that the composition $f \circ g: \Delta(\lambda-n \delta) \rightarrow X \rightarrow L(\lambda-n \delta)$ is surjective.

Now $L(\lambda-n \delta)$ is not a quotient of $V(\lambda)$ (since $n>0$ ), hence $X$ is a proper submodule of $V(\lambda)$. So its weights are strictly smaller than $\lambda$, i.e. $X$ is an object in $\mathcal{O}_{\Lambda}^{<\lambda}$. Since $P^{<\lambda}(\lambda-n \delta)$ is a projective cover of $L(\lambda-n \delta)$ in $\mathcal{O}_{\Lambda}^{<\lambda}$, there is a map $h: P^{<\lambda}(\lambda-n \delta) \rightarrow X$ such that the composition $P^{<\lambda}(\lambda-n \delta) \stackrel{h}{\rightarrow} X \stackrel{f}{\rightarrow} L(\lambda-n \delta)$ is surjective. We are going to show that the map $h$ factors over the quotient map $P^{<\lambda}(\lambda-n \delta) \rightarrow P^{\leqslant \lambda-n \delta}(\lambda-n \delta) \cong \Delta(\lambda-n \delta)$ so that we get an induced map $\Delta(\lambda-n \delta) \rightarrow X$, which we can take as the map $g$ that we wanted to construct.

By Lemma 6.5 the module $P^{<\lambda}(\lambda-n \delta)$ is an extension of its subquotients $P(\lambda-n \delta)_{[\nu]}$. Now $X$ is a submodule of $V(\lambda)$, which is a module of highest weight $\lambda$. So for each $r>0$ and $z \in \mathcal{A}_{r}$ we have $z^{V(\lambda)}=0$, hence $z^{X}=0$. Using the Propositions 6.11 and 6.14 and the assumption on the multiplicities, we can inductively show that each Verma subquotient of $P^{<\lambda}(\lambda-n \delta)$ lies in the kernel of $h$ except possibly $P(\lambda-n \delta)_{[\alpha \uparrow \lambda-n \delta]}$ and $P(\lambda-n \delta)_{[\lambda-n \delta]}$. But since $[X: L(\alpha \uparrow$ $\lambda-n \delta)]=0$ by part (1) of our main theorem, $P(\lambda-n \delta)_{[\alpha \uparrow \lambda-n \delta]}$ is also contained in the kernel, so we get an induced map

$$
P(\lambda-n \delta)^{\leqslant \lambda-n \delta} \cong \Delta(\lambda-n \delta) \rightarrow X,
$$

which is what we wanted to show. Hence we proved claim (1).

Secondly, we claim

(2) The map $\Delta(\lambda)_{\lambda-n \delta}^{\widehat{\mathfrak{n}}_{+}} \rightarrow V(\lambda)_{\lambda-n \delta}^{\widehat{\mathfrak{n}}^{+}}$that is induced by the canonical map $\pi: \Delta(\lambda) \rightarrow V(\lambda)$ is surjective.

So let $v \in V(\lambda)_{\lambda-n \delta}^{\widehat{\mathfrak{n}}^{+}}, v \neq 0$, and denote by $X$ the submodule of $V(\lambda)$ which is generated by $v$. Then $X$ is a highest weight module with highest weight $\lambda-n \delta$ and there is a short exact sequence

$$
0 \rightarrow \Delta(\alpha \downarrow \lambda) \rightarrow \pi^{-1}(X) \rightarrow X \rightarrow 0 .
$$

Now the Casimir element $C$ acts on $\pi^{-1}(X)$ as a scalar, since $\pi^{-1}(X)$ is a submodule of a Verma module. Hence we can apply Lemma 6.10 and we deduce that there is a submodule $Y$ of $\pi^{-1}(X)$ of highest weight $\lambda-n \delta$ that maps surjectively to $X$. In particular, there is a preimage of $v$ in $\pi^{-1}(X)_{\lambda-n \delta}^{\widehat{\mathfrak{n}}^{+}} \subset \Delta(\lambda)_{\lambda-n \delta}^{\widehat{\mathfrak{n}}_{+}}$. So we proved part (2).

Our next step is to prove

(3) We have $[\Delta(\alpha \downarrow \lambda): L(\lambda-n \delta)]=\operatorname{dim} \Delta(\alpha \downarrow \lambda)_{\lambda-n \delta}^{\widehat{\mathfrak{n}}_{+}}$.

Suppose that the claim is wrong, i.e. suppose that $[\Delta(\alpha \downarrow \lambda): L(\lambda-n \delta)]>$ $\operatorname{dim} \Delta(\alpha \downarrow \lambda)_{\lambda-n \delta}^{\widehat{\mathfrak{n}}_{+}}$. Let $f: \Delta(\alpha \downarrow \lambda) \rightarrow \Delta(\lambda)$ be a non-zero map and let us consider the composition $g: \Delta(\alpha \downarrow \lambda) \stackrel{f}{\rightarrow} \Delta(\lambda) \rightarrow \bar{\Delta}(\lambda)$. Let $K$ be the kernel and $X$ the image of $g$. Then $X$ is a highest weight module of highest weight $\alpha \downarrow \lambda$, so $[X: L(\alpha \downarrow \lambda)]=1$. As $g$ factors over the map $\Delta(\alpha \downarrow \lambda) \rightarrow \bar{\Delta}(\alpha \downarrow \lambda)$, part (1) of our main theorem implies $[X: L(\alpha \downarrow \lambda-l \delta)]=0$ for all $l>0$. Our induction assumption implies $[\bar{\Delta}(\lambda): L(\lambda-l \delta)]=0$ for all $0<l<n$, hence $[X: L(\lambda-l \delta)]=0$ for all $l<n$. 
Now the weights of $K$ are strictly smaller than $\alpha \downarrow \lambda$. By the induction assumption and part (1) of the main theorem, each subquotient of $K$ of type $L(\lambda-n \delta)$ hence corresponds to a singular vector of weight $\lambda-n \delta$. Together with the assumption $[\Delta(\alpha \downarrow \lambda): L(\lambda-n \delta)]>\operatorname{dim} \Delta(\alpha \downarrow \lambda)_{\lambda-n \delta}^{\widehat{\mathfrak{n}}_{+}}$, this allows us to deduce $[X: L(\lambda-n \delta)]>0$. So $\lambda-n \delta$ occurs as a maximal weight in the maximal submodule of $X$. Hence there is a primitive vector in $X$, hence in $\bar{\Delta}(\lambda)$, of weight $\lambda-n \delta$. But this contradicts Theorem 4.7. Hence our assumption $[\Delta(\alpha \downarrow \lambda): L(\lambda-n \delta)]>\operatorname{dim} \Delta(\alpha \downarrow \lambda)_{\lambda-n \delta}^{\widehat{\mathfrak{n}}_{+}}$leads to a contradiction, so we proved claim (3).

Now we can finish the proof of the theorem. Recall that we have to show that $[\Delta(\lambda): L(\lambda-n \delta)]=\operatorname{dim} \Delta(\lambda)_{\lambda-n \delta}^{\widehat{\mathfrak{n}}_{+}}$under the assumption that $[\Delta(\lambda): L(\lambda-l \delta)]=$ $\operatorname{dim} \Delta(\lambda)_{\lambda-l \delta}^{\widehat{\mathfrak{n}}_{+}}$for any $l<n$. We have

$$
\begin{aligned}
\operatorname{dim} \Delta(\lambda)_{\lambda-n \delta}^{\widehat{\mathfrak{n}}_{+}} & =\operatorname{dim} \Delta(\alpha \downarrow \lambda)_{\lambda-n \delta}^{\widehat{\mathfrak{n}}_{+}}+\operatorname{dim} V(\lambda)_{\lambda-n \delta}^{\widehat{\mathfrak{n}}_{+}} \quad \text { (by (2)) } \\
& =[\Delta(\alpha \downarrow \lambda): L(\lambda-n \delta)]+[V(\lambda): L(\lambda-n \delta)] \quad \text { (by (1) and (3)) } \\
& =[\Delta(\lambda): L(\lambda-n \delta)],
\end{aligned}
$$

which is what we wanted to prove.

\section{ACKNOWLEDGMENTS}

Both authors wish to thank the Emmy Noether Center in Erlangen, where parts of the research for this paper were done, for its hospitality and support. The second author wishes to thank Nara Women's University for its hospitality and the Landesstiftung Baden-Württemberg for supporting the project.

\section{REFERENCES}

[AJS94] Henning Haahr Andersen, Jens Carsten Jantzen, Wolfgang Soergel, Representations of quantum groups at a pth root of unity and of semisimple groups in characteristic $p$ : independence of $p$, Astérisque (1994), no. 220. MR.1272539 (95j:20036)

[Ara07] Tomoyuki Arakawa, Representation theory of W-algebras, Invent. Math. 169 (2) (2007), 219-320. MR2318558 (2009d:17039)

[AF09] Tomoyuki Arakawa, Peter Fiebig, The linkage principle for restricted critical level representations of affine Kac-Moody algebras, preprint arXiv:0909.4214.

[BD96] Alexander Beilinson, Vladimir Drinfeld, Quantization of Hitchin's fibration and Langlands' program, in Algebraic and geometric methods in mathematical physics (Kaciveli, 1993), volume 19 of Math. Phys. Stud., pages 3-7. Kluwer Acad. Publ., Dordrecht, 1996. MR1385674 (97m:14008)

[DGK82] Vinay Deodhar, Ofer Gabber, Victor Kac, Structure of some categories of representations of infinite-dimensional Lie algebras, Adv. Math.45 (1982), no. 1, 92-116. MR663417 (83i:17012)

[EF01] David Eisenbud, Edward Frenkel, Appendix to [Mus01], 2001.

[FBZ04] Edward Frenkel, David Ben-Zvi, Vertex algebras and algebraic curves, volume 88 of Mathematical Surveys and Monographs, American Mathematical Society, Providence, RI, second edition, 2004. MR2082709 (2005d:17035)

[Feř84] Boris Fergin, Semi-infinite homology of Lie, Kac-Moody and Virasoro algebras, Uspekhi Mat. Nauk, 39 (1984), no. 2(236), 195-196. MR740035 (85g:17003)

[FF90] Boris Feigin, Edward Frenkel, Representations of affine Kac-Moody algebras and bosonization, in Physics and mathematics of strings, 271-316. World Sci. Publ., Teaneck, NJ, 1990. MR1104262 (92d:17025) 
[FF92] - Affine Kac-Moody algebras at the critical level and Gel'fand-Dikiu algebras, in Infinite analysis, Part A, B (Kyoto, 1991), volume 16 of Adv. Ser. Math. Phys., 197-215. World Sci. Publ., River Edge, NJ, 1992. MR1187549 (93j:17049)

[Fie03] Peter Fiebig, Centers and translation functors for the category O over symmetrizable Kac-Moody algebras, Math. Z. 243 (2003), no. 4, 689-717. MR.1974579 (2004c:17051)

[Fie06] - The combinatorics of category $O$ for symmetrizable Kac-Moody algebras, Transform. Groups 11 (2006), no. 1, 29-49. MR2205072 (2006k:17040)

[Fie07] , Lusztig's conjecture as a moment graph problem; Bull. London Math. Soc. 42 (2010), no. 6, 957-972. MR2740015

[Fre07] Edward Frenkel, Langlands correspondence for loop groups, volume 103 of Cambridge Studies in Advanced Mathematics.Cambridge University Press, Cambridge, 2007. MR2332156 (2008h:22017)

[FG06] Edward Frenkel, Dennis Gaitsgory, Local geometric Langlands correspondence and affine Kac-Moody algebras, Algebraic geometry and number theory, Progr. Math., vol. 253, Birkhäuser Boston, Boston, MA, 2006, pp. 69-260. MR2263193 (2008e:17023)

[FG07] _ D-modules on the affine flag variety and representations of affine Kac-Moody algebras, Represent. Theory 13 (2009), 470-608. MR2558786

[Hay88] Takahiro Hayashi, Sugawara operators and Kac-Kazhdan conjecture, Invent. Math. 94 (1988), no. 1, 13-52. MR958588 (90c:17035)

[Jan79] Jens Carsten Jantzen, Moduln mit einem höchsten Gewicht, Lecture Notes in Mathematics 750, Springer, 1979. MR552943 (81m:17011)

[Kac90] Victor Kac, Infinite-dimensional Lie algebras, Third edition. Cambridge University Press, Cambridge, 1990. MR1104219 (92k:17038)

[Kac98]__ Vertex algebras for beginners, volume 10 of University Lecture Series. American Mathematical Society, Providence, RI, second edition, 1998. MR.1651389 (99f:17033)

[KK79] Victor Kac, David Kazhdan, Structure of representations with highest weight of infinitedimensional Lie algebras, Adv. Math. 34 (1979), 97-108. MR547842 (81d:17004)

[Kos78] Bertram Kostant, On Whittaker vectors and representation theory, Invent. Math. 48 (2) (1978),101-184. MR507800 (80b:22020)

[KT00] Masaki Kashiwara, Toshiyuki Tanisaki, Characters for irreducible modules with noncritical highest weights over affine Lie algebras, in: Wang, Jianpan (ed.) et al., Representations and quantizations. Proceedings of the international conference on representation theory, Shanghai, China, June 29-July 3, 1998. Beijing: China Higher Education Press (CHEP), 275-296 (2000). MR.1802178 (2001k:17037)

[Ku89] Jong Min Ku, Structure of the Verma module $M(-\rho)$ over Euclidean Lie algebras, J. Algebra 124 (1989), no. 2, 367-387. MR.1011602 (90k:17057)

[Li04] Haisheng Li, Vertex algebras and vertex Poisson algebras, Commun. Contemp. Math., 6(1) (2004), 61-110. MR.2048777 (2005d:17036)

[Lus80] George Lusztig, Hecke algebras and Jantzen's generic decomposition patterns, Adv. in Math. 37 (1980), no. 2, 121-164. MR591724 (82b:20059)

[Lus91] _ Intersection cohomology methods in representation theory, I. Satake (ed.), Proc. Internat. Congr. Math. Kyoto 1990, I, Springer (1991), 155-174. MR1159211 (93e:20059)

[Mal90] Feodor Malikov, Singular vectors corresponding to imaginary roots in Verma modules over affine Lie algebras, Math. Scand. 66 (1990), no. 1, 73-90. MR1060899 (91f:17024)

[Mat96] Olivier Mathieu, On some modular representations of affine Kac-Moody algebras at the critical level, Compositio Math. 102 (1996), no. 3, 305-312. MR1401425 (97g:17026)

[MP95] Robert Moody, Arturo Pianzola, Lie algebras with triangular decompositions, Canadian Mathematical Society Series of Monographs and Advanced Texts. A Wiley-Interscience Publication. John Wiley \& Sons, Inc., New York, 1995. MR.1323858 (96d:17025)

[Mus01] Mircea Mustaţă, Jet schemes of locally complete intersection canonical singularities, Invent. Math. 145 (3) (2001), 397-424. With an appendix by David Eisenbud and Edward Frenkel. MR1856396 (2002f:14005)

[RCW82] Alvany Rocha-Caridi, Nolan Wallach, Projective modules over graded Lie algebras, Mathematische Zeitschrift 180 (1982), 151-177. MR661694 (83h:17018)

[Soe90] Wolfgang Soergel, Kategorie $\mathcal{O}$, perverse Garben, und Moduln über den Koinvarianten zur Weylgruppe, J. Am. Math. Soc. 3 (1990), no. 2, 421-445 MR1029692 (91e:17007) 
[Soe97] _ Kazhdan-Lusztig-Polynome und eine Kombinatorik für Kipp-Moduln, Represent. Theory 1 (1997), 37-68 (electronic). MR.1445511 (99d:17023)

Research Institute for Mathematical Sciences, Kyoto University, Kyoto 606-8502, JAPAN

E-mail address: arakawa@kurims.kyoto-u.ac.jp

Department Mathematik, Universität Erlangen-Nürnberg, 91058 Erlangen, Germany

E-mail address: fiebig@mi.uni-erlangen.de 